\title{
DENSITY CONDITIONS FOR PANCHROMATIC COLOURINGS OF HYPERGRAPHS
}

\author{
ALEXANDR V. KOSTOCHKA*, DOUGLAS R. WOODALL
}

Received November 10, 1998

Let $\mathcal{H}=(V, \mathcal{E})$ be a hypergraph. A panchromatic $t$-colouring of $\mathcal{H}$ is a $t$-colouring of its vertices such that each edge has at least one vertex of each colour; and $\mathcal{H}$ is panchromatically $t$-choosable if, whenever each vertex is given a list of $t$ colours, the vertices can be coloured from their lists in such a way that each edge receives at least $t$ different colours. The Hall ratio of $\mathcal{H}$ is $h(\mathcal{H})=\min \{|\bigcup \mathcal{F}| /|\mathcal{F}|: \emptyset \neq \mathcal{F} \subseteq \mathcal{E}\}$. Among other results, it is proved here that if every edge has at least $t$ vertices and $|\bigcup \mathcal{F}| \geqslant(t-1)|\mathcal{F}|-t+3$ whenever $\emptyset \neq \mathcal{F} \subseteq \mathcal{E}$, then $\mathcal{H}$ is panchromatically $t$-choosable, and this condition is sharp; the minimum $c_{t}$ such that every $t$-uniform hypergraph with $h(\mathcal{H})>c_{t}$ is panchromatically $t$-choosable satisfies $t-2+3 /(t+1) \leqslant c_{t} \leqslant t-2+4 /(t+2)$; and except possibly when $t=3$ or 5 , a $t$-uniform hypergraph is panchromatically $t$-colourable if $|\bigcup \mathcal{F}| \geqslant\left(\left(t^{2}-2 t+2\right)|\mathcal{F}|+t-1\right) / t$ whenever $\emptyset \neq \mathcal{F} \subseteq \mathcal{E}$, and this condition is sharp. This last result dualizes to a sharp sufficient condition for the chromatic index of a hypergraph to equal its maximum degree.

\section{Introduction}

Let $\mathcal{H}=(V, \mathcal{E})$ be a hypergraph with vertex-set $V(\mathcal{H})=V$ and edge-set $\mathcal{E}(\mathcal{H})=\mathcal{E}$. A panchromatic t-colouring of $\mathcal{H}$ is a function $f: V \rightarrow C$, where $C$ is a set of $t$ colours, such that each edge contains at least one vertex of each colour. If each vertex of $\mathcal{H}$ is assigned a list of $t$ colours, then a panchromatic t-list-colouring of $\mathcal{H}$ is a colouring in which each vertex is given a colour from

Mathematics Subject Classification (2000): 05C65, 05C15, 05C35

* This work was carried out while the first author was visiting Nottingham, funded by Visiting Fellowship Research Grant GR/L54585 from the Engineering and Physical Sciences Research Council. The work of this author was also partly supported by grants 96-01-01614 and 97-01-01075 of the Russian Foundation for Fundamental Research. 
its own list and each edge contains vertices with at least $t$ different colours. We say that $\mathcal{H}$ is panchromatically $t$-choosable or, equivalently, panchromatically t-list-colourable, if it has a panchromatic $t$-list-colouring whenever a list of $t$ colours is assigned to each vertex of $\mathcal{H}$; clearly this implies that $\mathcal{H}$ is panchromatically $t$-colourable. The connection between panchromatic colourings and edge-colourings of the dual hypergraph is illustrated in Theorem 3 below.

The Hall ratio of $\mathcal{H}$ is $h(\mathcal{H}):=\min \{|\bigcup \mathcal{F}| /|\mathcal{F}|: \emptyset \neq \mathcal{F} \subseteq \mathcal{E}\}$, where we write $\cup \mathcal{F}$ as a shorthand for $\bigcup_{F \in \mathcal{F}} F$. The Hall ratio is so called because Hall's theorem [4] says that $\mathcal{H}$ has a system of distinct representatives if and only if $h(\mathcal{H}) \geqslant 1$. For a graph $G$, the Hall ratio $h(G)$ is related to the maximum average degree $\operatorname{mad}(G)$ over all subgraphs of $G$ by $\operatorname{mad}(G)=2 / h(G)$; thus the obvious inequality $\operatorname{mad}(G) \geqslant \chi(G)-1$ can be rewritten as $h(G) \leqslant 2 /(\chi(G)-1)$, and results of, for example, Gallai [3] and Krivelevich [5] giving lower bounds on $\operatorname{mad}(G)$ for a $k$-critical graph $G$, can be reformulated similarly in terms of $h(G)$. For hypergraphs in general, it is well known $[6,8,7,1]$ that if $h(\mathcal{H})>1$ then $\mathcal{H}$ is (panchromatically) 2-colourable, and Lovász [6] proved the more general result that if $h(\mathcal{H})>t-1$ then $\mathcal{H}$ is panchromatically $t$-colourable.

In Section 2, we generalize this result from colourability to choosability. For $t \geqslant 3$, we shall see in Theorem 5 that if $h(\mathcal{H})>t-1$, or if $h(\mathcal{H}) \geqslant t-1$ and every edge has at least $t$ vertices, then $\mathcal{H}$ is panchromatically $t$-choosable. In Theorem 7 we prove the marginally stronger result that if every edge has at least $t$ vertices and $|\cup \mathcal{F}| \geqslant(t-1)|\mathcal{F}|-t+3$ whenever $\emptyset \neq \mathcal{F} \subseteq \mathcal{E}$, then $\mathcal{H}$ is panchromatically $t$-choosable, and this condition on $|\bigcup \mathcal{F}|$ is sharp even for panchromatic $t$-colourability.

In Section 3 we consider the panchromatic choosability of $t$-uniform hypergraphs. If $\mathcal{H}$ is $t$-uniform (that is, every edge contains exactly $t$ vertices) then the above condition can be weakened somewhat, as we described in the Abstract and as we shall see in Theorem 9.

In Section 4 we consider the analogous problem for colourability rather than choosability. Here we can do better, and we prove the following result.

Theorem 1. Let $t \geqslant 2, t \notin\{3,5\}$, and let $\mathcal{H}=(V, \mathcal{E})$ be a $t$-uniform hypergraph such that

$$
|\bigcup \mathcal{F}| \geqslant \frac{\left(t^{2}-2 t+2\right)|\mathcal{F}|+t-1}{t} \quad \text { whenever } \quad \emptyset \neq \mathcal{F} \subseteq \mathcal{E}
$$

Then $\mathcal{H}$ is panchromatically $t$-colourable. For $t \in\{3,5\}$, the same conclusion follows if the final -1 in (1.1) is omitted. 
This bound is sharp if $t \notin\{3,5\}$. We conjecture that these exceptions in Theorem 1 are unnecessary, and that the condition even suffices to ensure choosability rather than colourability:

Conjecture 2. Let $t \geqslant 2$. If a $t$-uniform hypergraph $\mathcal{H}=(V, \mathcal{E})$ satisfies (1.1), then it is panchromatically $t$-choosable.

The only value of $t$ for which we know this conjecture to be true is $t=2$, for which the result follows from Theorem 5 .

Duchet [2] defines a hypergraph to have the edge-colouring property if its chromatic index is equal to its maximum degree, and he quotes some sufficient conditions for a hypergraph to have this property. Theorem 1 can be reformulated so as to give a different type of condition for this property. Note first that, by Theorem 1, (1.1) suffices to ensure that every hypergraph $\mathcal{H}$ (not necessarily $t$-uniform) with maximum edge size $t$ is strictly $t$-colourable; that is, its vertices can be coloured with $t$ colours in such a way that no two vertices in the same edge have the same colour. This is because if there is an edge containing $s<t$ vertices, then adding $t-s$ vertices of degree 1 into this edge will not cause (1.1) to fail, nor create a strict $t$-colouring if one did not exist already; thus the result for $t$-uniform hypergraphs implies the more general result. But a strict colouring of $\mathcal{H}$ corresponds to a proper edgecolouring of the dual hypergraph $\mathcal{H}^{*}$, and the maximum edge size of $\mathcal{H}$ is the maximum degree of $\mathcal{H}^{*}$. Thus Theorem 1 is equivalent to the following.

Theorem 3. Let $t \geqslant 2, t \notin\{3,5\}$, and let $\mathcal{H}^{*}=\left(V^{*}, \mathcal{E}^{*}\right)$ be a hypergraph with maximum degree at most $t$. Suppose that, for every $k\left(1 \leqslant k \leqslant\left|V^{*}\right|\right)$, every set of $k$ vertices is incident with at least $\left(\left(t^{2}-2 t+2\right) k+t-1\right) / t$ edges. Then $\mathcal{H}^{*}$ is edge-t-colourable.

This bound, which is sharp since the bound in Theorem 1 is sharp, seems somewhat unusual.

Throughout the paper, $\mathbb{N}$ denotes the set of positive integers. As usual, the degree of a vertex in a hypergraph is the number of edges containing it, and two vertices are adjacent if there is an edge containing both of them. A $d$-vertex is a vertex with degree $d$. A vertex is essential if its degree is at least 2 , and inessential otherwise.

\section{Panchromatic choosability of hypergraphs}

In this section we consider general (not necessarily uniform) hypergraphs. We begin with the extremal example. 
Example 4. Let $\mathcal{H}=\mathcal{H}(s, t)$ be the hypergraph with vertices $v_{0}$ and $v_{i, j}$ $(1 \leqslant i \leqslant s, 2 \leqslant j \leqslant t)$ and edges $A_{i}:=\left\{v_{0}, v_{i, 2}, \ldots, v_{i, t}\right\} \quad(1 \leqslant i \leqslant s)$ and $A_{0}:=\left\{v_{1, t}, v_{2, t}, \ldots, v_{s, t}\right\}$. Then $\mathcal{H}$ has no panchromatic $t$-colouring, since no element of $A_{0}$ can be given the same colour as $v_{0}$. But $\mathcal{H}$ has $s+1$ edges and $s(t-1)+1$ vertices, and $|\bigcup \mathcal{F}| \geqslant(t-1)|\mathcal{F}|-t+2$ for all $\mathcal{F} \subseteq \mathcal{E}(\mathcal{H})$, with strict inequality unless $\mathcal{F}=\mathcal{E}(\mathcal{H})$. And $h(\mathcal{H})=(s(t-1)+1) /(s+1)$, which tends to $t-1$ from below as $s \rightarrow \infty$, so that to require $h(\mathcal{H})>t-1-\epsilon$ would not suffice to ensure panchromatic $t$-colourability.

We now prove the best possible result involving the Hall ratio. The sufficiency of the conditions follows from Theorem 7 , but we include a direct proof here since it is relatively short and so that we can cite it as part of the proof of Theorem 7 .

Theorem 5. Let $\mathcal{H}$ be a hypergraph and $t \in \mathbb{N}$. If (i) $h(\mathcal{H})>t-1$, or (ii) $t \geqslant 3$, every edge of $\mathcal{H}$ has at least $t$ vertices and $h(\mathcal{H}) \geqslant t-1$, then $\mathcal{H}$ is panchromatically $t$-choosable; moreover, these conditions on $h(\mathcal{H})$ are sharp, even for panchromatic t-colourability.

Proof. Condition (i) is clearly sharp, since $h(\mathcal{H}) \geqslant t-1$ is not enough to ensure that every edge has at least $t$ vertices, which is necessary for the existence of a panchromatic $t$-colouring. Example 4 shows that condition (ii) is sharp. The odd cycles show that condition (ii) would not suffice to ensure panchromatic $t$-colourability if $t=2$ (although it does suffice, rather trivially, if $t=1$ ).

We now prove that the conditions are sufficient. Let $\mathcal{E}(\mathcal{H})=\left\{A_{1}, \ldots, A_{m}\right\}$. A well-known generalization of Hall's theorem (the harem theorem) says that the condition $h(H) \geqslant t-1$ is necessary and sufficient for the existence of disjoint sets $B_{1}, \ldots, B_{m}$ such that $B_{i} \subseteq A_{i}$ and $\left|B_{i}\right|=t-1$ for each $i$. So suppose that such sets $B_{i}$ exist. Suppose that every vertex is given a list of $t$ colours. Colour first every vertex that is in no set $B_{i}$, giving each vertex an arbitrary colour from its list. If possible, choose a set $A_{i}$ that contains a coloured vertex but such that all vertices in $B_{i}$ are uncoloured, and colour all vertices of $B_{i}$ from their lists in such a way that $A_{i}$ contains vertices of $t$ different colours; clearly this is possible. Repeat until no such set $A_{i}$ remains. Now let $K$ be the set of indices $i$ such that $B_{i}$ is uncoloured. Then $\left|\bigcup_{i \in K} A_{i}\right|=\left|\bigcup_{i \in K} B_{i}\right|=(t-1)|K|$, and so if $h(\mathcal{H})>t-1$ then $K=\emptyset$ and the colouring is complete.

Now suppose $K \neq \emptyset, t \geqslant 3$, every edge has at least $t$ vertices, and $h(\mathcal{H})=$ $t-1$. For each $i \in K$, choose a vertex $v_{i} \in A_{i} \backslash B_{i}$ and consider the digraph $D$ with the $v_{i}$ as vertices in which there is an arc from $v_{i}$ to $v_{j}$ whenever $v_{i} \in B_{j}$. (Note that $D$ may have fewer than $|K|$ vertices, since it is possible 
that $v_{j}=v_{k}$ when $j \neq k$. In this case, the above definition implies that there is an arc from $v_{i}$ to $v_{j}=v_{k}$ whenever $v_{i} \in B_{j} \cup B_{k}$.) Then each vertex of $D$ has outdegree 1 . Since $t \geqslant 3$, it is possible to colour the vertices of $D$ from their lists in such a way that not only do adjacent vertices get different colours, but no set $B_{i}$ contains two vertices of the same colour. (Colour first the vertices of directed cycles in $D$, then go back up the branches of any trees rooted at such vertices, colouring as you go.) Note that each set $A_{i}$ $(i \in K)$ now has at least one coloured vertex $v_{i}$, and may have other coloured vertices in $B_{i}$, but it does not contain two vertices of the same colour. So we may colour the remaining vertices in $B_{i}$ from their lists so that $A_{i}$ contains vertices of $t$ different colours. All vertices of $\mathcal{H}$ are now coloured, and we have a panchromatic $t$-colouring.

Although Theorem 5 is sharp, we can improve it by changing the nature of the condition slightly. We do this first for $t$-uniform hypergraphs, although we should note that for such hypergraphs the result stated here is not sharp; the question of how much it can be improved forms the subject of the next section.

Lemma 6. Let $t \in \mathbb{N}$ and let $\mathcal{H}=(V, \mathcal{E})$ be a $t$-uniform hypergraph such that $|\cup \mathcal{F}| \geqslant(t-1)|\mathcal{F}|-t+3$ whenever $\emptyset \neq \mathcal{F} \subseteq \mathcal{E}$. Let each vertex of $\mathcal{H}$ be assigned a list of $t$ colours, let $A$ be any edge of $\mathcal{H}$, and let each vertex of $A$ be coloured from its list in such a way that no two vertices of $A$ have the same colour. Then this colouring of $A$ can be extended to a panchromatic t-list-colouring of $\mathcal{H}$.

Proof. We prove the result by induction on $t$, noting that it is true if $t=1$ even without the condition on $|\cup \mathcal{F}|$. So suppose $t \geqslant 2$, and let $\mathcal{H}$ if possible be a counterexample with as few edges as possible, and with lists of colours assigned so that the required colouring cannot be obtained. Clearly $\mathcal{H}$ is connected.

Suppose first there is a proper subset $\mathcal{J} \subset \mathcal{E}$ such that $|\bigcup \mathcal{J}|=(t-1)|\mathcal{J}|-$ $t+3$. Choose $\mathcal{J}$ to be a largest proper subset of $\mathcal{E}$ with this property. Since $\mathcal{H}$ is connected, there is an edge $E$ in $\mathcal{E} \backslash \mathcal{J}$ that intersects $\cup \mathcal{J}$, and if $\mathcal{F}:=\mathcal{J} \cup\{E\}$ then $|\cup \mathcal{F}|=(t-1)|\mathcal{F}|-t+3$ (since $\geqslant$ holds by hypothesis, and $>$ is clearly impossible). Since $\mathcal{F}$ cannot therefore be a proper subset of $\mathcal{E}$, by the choice of $\mathcal{J}$, it follows that $\mathcal{F}=\mathcal{E}$. If $A \in \mathcal{J}$, we can extend the colouring of $A$ to a panchromatic $t$-list-colouring of $(\cup \mathcal{J}, \mathcal{J})$ by the choice of $\mathcal{H}$, since $\mathcal{J}$ has fewer edges than $\mathcal{H}$, and this colouring is then easily extended panchromatically to the remaining $t-1$ vertices of $E$. If however $A=E$, then choose an edge $F \in J$ such that $A \cap F \neq \emptyset$, extend the colouring of $A \cap F$ panchromatically to the remaining $t-1$ vertices of $F$, and then 
extend this colouring of $F$ to a panchromatic $t$-list-colouring of $\mathcal{J}$. In either case, we have extended the colouring of $A$ to a panchromatic $t$-list-colouring of $\mathcal{H}$, and this contradiction shows that in fact $|\bigcup \mathcal{J}|>(t-1)|\mathcal{J}|-t+3$ for every proper subset $\mathcal{J} \subset \mathcal{E}$.

Suppose now that some edge $E$ is contained in the union of all the other edges. If $\mathcal{F}:=\mathcal{E} \backslash\{E\}$, then $|\bigcup \mathcal{F}| \geqslant(t-1)(|\mathcal{F}|+1)-t+3=(t-1)|\mathcal{F}|+2$. This means that $\mathcal{H}-E$ is not connected; that is, one can write $\mathcal{E}=\{E\} \cup \mathcal{E}_{1} \cup \mathcal{E}_{2}$ in such a way that no edge of $\mathcal{E}_{1}$ has any vertices in common with any edge of $\mathcal{E}_{2}$. Without loss of generality, $A \in\{E\} \cup \mathcal{E}_{1}$. Since $\mathcal{H}$ was a counterexample with as few edges as possible, we can extend the given colouring of $A$ to a panchromatic $t$-list-colouring of $\{E\} \cup \mathcal{E}_{1}$, and then extend the resulting colouring of $E$ to a panchromatic $t$-list-colouring of $\{E\} \cup \mathcal{E}_{2}$. This gives a panchromatic $t$-list-colouring of $\mathcal{H}$, which is a contradiction. Thus each edge $E$ contains a vertex that is in no other edge; choose such a vertex $v_{E}$ for each edge $E$.

Finally, let $w$ be a vertex in $A$ that is contained in at least one other edge (which must exist, since $\mathcal{H}$ is connected). For each edge $E$, let $E^{\prime}:=E \backslash\{w\}$ if $w \in E$ and let $E^{\prime}:=E \backslash\left\{v_{E}\right\}$ otherwise. Let $c$ be a colour in the list of $w$. Consider the $(t-1)$-uniform hypergraph $\mathcal{H}^{\prime}$ with edge-set $\left\{E^{\prime}: E \in \mathcal{E}\right\}$, with colour $c$ removed from all lists. Then $\mathcal{H}^{\prime}$ satisfies the hypotheses of the theorem with $t-1$ in place of $t$, because $\left|\bigcup_{E \in \mathcal{F}} \mathcal{F}^{\prime}\right| \geqslant|\bigcup \mathcal{F}|-|\mathcal{F}|$ for each $\mathcal{F} \subseteq \mathcal{E}$, with strict inequality when $\mathcal{F}=\mathcal{E}$, so that $\left|\bigcup_{E \in \mathcal{F}} \mathcal{F}^{\prime}\right| \geqslant(t-2)|\mathcal{F}|-t+4$ for all $\mathcal{F} \subseteq \mathcal{E}$. Now the given colouring of $A^{\prime}$ can first be extended to a panchromatic $(t-1)$-list-colouring of $\mathcal{H}^{\prime}$ by the induction hypothesis, and then we can colour $w$ with $c$ and colour each removed vertex $v_{E}$ with a colour from its list so as to obtain a panchromatic $t$-list-colouring of $\mathcal{H}$. This contradiction completes the proof.

We now extend Lemma 6 to nonuniform hypergraphs, and simultaneously prove a result about the critical hypergraphs. A hypergraph $\mathcal{H}$ is critically panchromatically non-t-colourable if $\mathcal{H}$ has no isolated vertices and $\mathcal{H}$ is not panchromatically $t$-colourable, but every hypergraph obtained from $\mathcal{H}$ by deleting an edge is panchromatically t-colourable. A critical panchromatically non-t-choosable hypergraph is defined analogously.

Theorem 7. Let $t \in \mathbb{N}$ and let $\mathcal{H}=(V, \mathcal{E})$ be a hypergraph in which every edge has at least $t$ vertices.

(a) If $|\bigcup \mathcal{F}| \geqslant(t-1)|\mathcal{F}|-t+3$ whenever $\emptyset \neq \mathcal{F} \subseteq \mathcal{E}$, then $\mathcal{H}$ is panchromatically $t$-choosable, and this condition on $|\bigcup \mathcal{F}|$ is sharp even for panchromatic $t$ colourability (except when $t=1$ ).

(b) If $t \geqslant 3$ and $\mathcal{H}$ is critically panchromatically non-t-colourable, or critically panchromatically non-t-choosable, then $|\cup \mathcal{E}| \leqslant(t-1)|\mathcal{E}|-t+2$. 
Proof. Example 4 shows that the condition in (a) is sharp when $t \geqslant 2$. The rest of (a) follows from Theorem 5 if $t \leqslant 2$ and from (b) if $t \geqslant 3$, and so it would suffice to prove (b). However, it is more convenient to show first that (b) follows from (a), and then prove (a).

Suppose if possible that $\mathcal{H}$ is a counterexample to (b), and suppose that, if $\mathcal{H}$ is critically panchromatically non- $t$-choosable, then each vertex of $\mathcal{H}$ is given a list of $t$ colours for which $\mathcal{H}$ is not panchromatically $t$-list-colourable. Choose $s$ maximal such that

$$
|\bigcup \mathcal{F}| \geqslant(t-1)|\mathcal{F}|-t+s \quad \text { whenever } \emptyset \neq \mathcal{F} \subseteq \mathcal{E},
$$

and let $\mathcal{J}$ be any subset of $\mathcal{E}$ for which

$$
|\bigcup \mathcal{J}|=(t-1)|\mathcal{J}|-t+s
$$

Suppose $\mathcal{J} \neq \mathcal{E}$. Then $(\bigcup \mathcal{J}, \mathcal{J})$ is panchromatically $t$-colourable or $t$ choosable, as appropriate, by the criticality of $\mathcal{H}$. If $\mathcal{F}^{\prime} \subseteq \mathcal{E} \backslash \mathcal{J}$ then, taking $\mathcal{F}:=\mathcal{F}^{\prime} \cup \mathcal{J}$ in (2.1), and using (2.2), we find that $\left|\cup \mathcal{F}^{\prime} \backslash \cup \mathcal{J}\right| \geqslant(t-1)\left|\mathcal{F}^{\prime}\right|$. Since this holds for all $\mathcal{F}^{\prime} \subseteq \mathcal{E} \backslash \mathcal{J}$, the argument in the last paragraph of the proof of Theorem 5 shows that any panchromatic $t$-colouring of $(\bigcup \mathcal{J}, \mathcal{J})$ can be extended to a panchromatic $t$-colouring of $\mathcal{H}$ in which, if appropriate, each vertex of $V \backslash \cup \mathcal{J}$ receives a colour from its list. This contradiction shows that $\mathcal{J}=\mathcal{E}$.

It remains to prove that $s \leqslant 2$. This will follow from (a), which says that if $s \geqslant 3$ then $\mathcal{H}$ is panchromatically $t$-choosable.

So let $\mathcal{H}$ now be a counterexample to (a) with $t \geqslant 3$ for which $\sum_{E \in \mathcal{E}}|E|$ is as small as possible, and assume that each vertex of $\mathcal{H}$ is given a list of $t$ colours. Clearly $\mathcal{H}$ is connected. Define $s$ to be maximal such that (2.1) holds, so that $s \geqslant 3$ by hypothesis, and note that if (2.2) holds then the above argument shows that $\mathcal{J}=\mathcal{E}$. Suppose some edge $E \in \mathcal{E}$ contains more than $t$ vertices. Since $\mathcal{H}$ is connected, $E$ contains a vertex $v$ that belongs to another edge as well. Since we have just seen that strict inequality holds in (2.1) except when $\mathcal{F}=\mathcal{E}$, removing $v$ from $E$ will not violate any of the hypotheses of (a), nor will it make the hypergraph panchromatically $t$-choosable, and so the resulting hypergraph will violate the choice of $\mathcal{H}$ as a counterexample for which $\sum_{E \in \mathcal{E}}|E|$ is as small as possible. This contradiction shows that $\mathcal{H}$ is $t$-uniform, and the result now follows from Lemma 6 .

\section{Panchromatic choosability of $t$-uniform hypergraphs}

Theorems 5 and 7 are not sharp for $t$-uniform hypergraphs, as we shall see in Theorem 9. We first give what we conjecture to be the extremal example. (If $t=2$ then this example is an odd cycle, and it is certainly extremal.) 
Example 8. For $t \geqslant 2$, let $\mathcal{H}^{(t)}$ be the $t$-uniform hypergraph with vertices $v_{0}, v$ and $v_{i, j}(1 \leqslant i \leqslant t-1,2 \leqslant j \leqslant t)$, whose edges are $\left\{v_{0}, v_{i, 2}, \ldots, v_{i, t}\right\}$ $(1 \leqslant i \leqslant t-1)$ and $\left\{v_{1, t}, v_{2, t}, \ldots, v_{t-1, t}, v\right\}$. Then $\mathcal{H}^{(t)}$ has $t^{2}-2 t+3$ vertices and $t$ edges; and in any panchromatic $t$-colouring of $\mathcal{H}^{(t)}, v$ must be given the same colour as $v_{0}$. Call $\mathcal{H}^{(t)}$ a superlink joining $v_{0}$ and $v$.

Now, for $s \geqslant 1$, form a $t$-uniform hypergraph $\mathcal{H}_{s}^{(t)}$ by taking vertices $v_{0}, v_{1}, \ldots, v_{s}$ and adding superlinks joining $v_{i-1}$ and $v_{i}(1 \leqslant i \leqslant s)$ and an ordinary edge (set of $t$ vertices) containing $v_{s}$ and $v_{0}$, these $s$ superlinks and one edge having no vertices in common except for $v_{0}, v_{1}, \ldots, v_{s}$. Then $\mathcal{H}_{s}^{(t)}$ is not panchromatically $t$-colourable, and it has $s\left(t^{2}-2 t+2\right)+(t-1)$ vertices and $s t+1$ edges. So if its edge-set is $\mathcal{E}$ then

$$
|\bigcup \mathcal{E}|=s\left(t^{2}-2 t+2\right)+(t-1)=\frac{\left(t^{2}-2 t+2\right)|\mathcal{E}|+t-2}{t}
$$

and

$$
|\bigcup \mathcal{F}|>\frac{\left(t^{2}-2 t+2\right)|\mathcal{F}|+t-2}{t} \quad \text { whenever } \quad \emptyset \neq \mathcal{F} \varsubsetneqq \mathcal{E} .
$$

In particular, $\mathcal{H}_{1}^{(t)}$ is a $t$-uniform hypergraph that is not panchromatically $t$-colourable and for which

$$
h\left(H_{1}^{(t)}\right)=\frac{t^{2}-t+1}{t+1}=t-2+\frac{3}{t+1},
$$

the lower bound for $c_{t}$ stated in the Abstract.

We now prove a simple upper bound for $c_{t}$. Recall that a $d$-vertex is a vertex with degree $d$, and a vertex is essential if its degree is at least 2 .

Theorem 9. If $t \geqslant 2$ and $\mathcal{H}=(V, \mathcal{E})$ is a $t$-uniform hypergraph with $h(\mathcal{H})>$ $t-2+4 /(t+2)$, then $\mathcal{H}$ is panchromatically $t$-choosable.

Proof. There is no loss of generality in supposing that $\mathcal{H}$ has no isolated vertices. Suppose that $\mathcal{H}$, with a list of $t$ colours on each vertex, is a counterexample to the theorem with no isolated vertices, with as few edges as possible, and, subject to these conditions, with as many vertices as possible. We first prove two claims.

Claim 1. No edge can contain $t-1$ or more inessential vertices.

Proof. If $E$ is such an edge, then the hypergraph $\mathcal{H}^{\prime}$ obtained from $\mathcal{H}$ by deleting $E$ and all its 1 -vertices is panchromatically $t$-choosable, by the minimality of $\mathcal{H}$ as a counterexample, and a panchromatic $t$-colouring of $\mathcal{H}^{\prime}$ can clearly be extended to $\mathcal{H}$ by colouring each 1-vertex of $E$ in turn from its own list. This contradiction proves Claim 1. 
Claim 2. Every essential vertex is adjacent to at least $t$ other essential vertices.

Proof. If $v$ is an essential vertex that is adjacent to at most $t-1$ other essential vertices, let $\mathcal{H}^{\prime \prime}$ be a hypergraph obtained from $\mathcal{H}$ by splitting $v$ into two vertices $v^{\prime}, v^{\prime \prime}$, one of which is contained in one edge of $\mathcal{H}$ that contained $v$ and the other of which is contained in all the other edges that contained $v$. Let $v^{\prime}$ and $v^{\prime \prime}$ be given arbitrary lists of $t$ colours. Since $\mathcal{H}^{\prime \prime}$ has the same number of edges as $\mathcal{H}$ but more vertices, it is panchromatically $t$-choosable. Given a panchromatic $t$-colouring of $\mathcal{H}^{\prime \prime}$, we obtain a panchromatic $t$-colouring of $\mathcal{H}$ as follows. Give every essential vertex of $\mathcal{H}$ that occurs in $\mathcal{H}^{\prime \prime}$ the same colour as in $\mathcal{H}^{\prime \prime}$. Give $v$ a colour from its list that is different from the colours of all the essential vertices adjacent to it, which is possible since there are at most $t-1$ such essential vertices. Finally, give each 1 -vertex in turn a colour from its list that is different from the colours of all already-coloured vertices in its one edge. This shows that $\mathcal{H}$ is panchromatically $t$-choosable, and this contradiction completes the proof of Claim 2.

Now suppose that each vertex of $\mathcal{H}$ is allocated a charge equal to its degree, and that each essential vertex then redistributes $1 / t$ units of its charge to each 1 -vertex adjacent to it. Since every edge contains at least two essential vertices by Claim 1 , every 1 -vertex ends up with at least $1+2 / t$ units of charge. A vertex with degree $d \geqslant 2$ is adjacent to at most $d(t-1)-t$ inessential vertices by Claim 2 , and so ends up with at least $d-d(1-1 / t)+1=$ $1+d / t \geqslant 1+2 / t$ units of charge. Note that the total charge allocated was $t|\mathcal{E}|$, the total charge after the redistribution is at least $(1+2 / t)|V|$, and no charge was created or destroyed in the redistribution. Therefore $(t+2)|V| \leqslant t^{2}|\mathcal{E}|$ and

$$
\frac{|\cup \mathcal{E}|}{|\mathcal{E}|}=\frac{|V|}{|\mathcal{E}|} \leqslant \frac{t^{2}}{t+2}=t-2+\frac{4}{t+2}
$$

contrary to the hypothesis of the theorem. This contradiction completes the proof.

\section{Panchromatic colourings of $t$-uniform hypergraphs}

In this section we consider the colourability, rather than the choosability, of $t$-uniform hypergraphs. Let $\mathcal{H}=(V, \mathcal{E})$ be such a hypergraph. If $W \subseteq V$, let $\mathcal{E}(W)=\mathcal{E}_{\mathcal{H}}(W)$ be the set, and $e(W)=e_{\mathcal{H}}(W)$ be the number, of edges of $\mathcal{H}$ contained in $W$. Define the surplus of $W$ to be

$$
\operatorname{sur}(W)=\operatorname{sur}_{\mathcal{H}}(W)=t|W|-\left(t^{2}-2 t+2\right) e_{\mathcal{H}}(W)-t+2 .
$$


Then the following two conditions are equivalent:

$$
\begin{gathered}
\operatorname{sur}_{\mathcal{H}}(W) \geqslant 1 \text { whenever } \emptyset \neq W \subseteq V ; \\
|\cup \mathcal{F}| \geqslant \frac{\left(t^{2}-2 t+2\right)|\mathcal{F}|+t-1}{t} \text { whenever } \emptyset \neq \mathcal{F} \subseteq \mathcal{E} .
\end{gathered}
$$

For, if (4.2) holds then (4.3) follows on setting $W=\bigcup \mathcal{F}$ (so that $e_{\mathcal{H}}(W) \geqslant$ $|\mathcal{F}|$ ); and if (4.3) holds then (4.2) follows on setting $\mathcal{F}=\mathcal{E}_{\mathcal{H}}(W)$ (so that $|W| \geqslant|\bigcup \mathcal{F}|)$. We shall say that a $t$-uniform hypergraph $\mathcal{H}=(V, \mathcal{E})$ is well spread if it satisfies (4.2) (or, equivalently, (4.3)).

The rest of this paper is devoted to the proof of the following theorem.

Theorem 10. Let $t \geqslant 2, t \notin\{3,5\}$, and let $\mathcal{H}=(V, \mathcal{E})$ be a well-spread $t$-uniform hypergraph. Then $\mathcal{H}$ is panchromatically $t$-colourable.

Note that (4.3) is the same as (1.1). Thus Theorem 10 contains all but the last sentence of Theorem 1, and is best possible by Example 8, except for the annoying fact that we have been unable to prove the result if $t=3$ or 5 : the lemmas all hold for these values of $t$, but they seem inadequate to prove the result (although the conclusion holds under the stronger hypothesis $\operatorname{sur}_{\mathcal{H}}(W) \geqslant 2$ ). We conjecture that the result holds for these values too. Note that if $t=2$ then $(4.3)$ says that $h(\mathcal{H})>1$, and so the result follows from Theorem 5 . Thus from now on we shall assume $t \geqslant 3$.

The following property is straightforward.

Lemma 11. For each two subsets $U, W \subseteq V(\mathcal{H})$,

$$
\operatorname{sur}_{\mathcal{H}}(U \cup W)+\operatorname{sur}_{\mathcal{H}}(U \cap W) \leqslant \operatorname{sur}_{\mathcal{H}}(U)+\operatorname{sur}_{\mathcal{H}}(W) .
$$

Proof. Since $|U \cup W|+|U \cap W|=|U|+|W|$, this is equivalent to saying that

$$
e_{\mathcal{H}}(U \cup W)+e_{\mathcal{H}}(U \cap W) \geqslant e_{\mathcal{H}}(U)+e_{\mathcal{H}}(W) .
$$

This holds because $e_{\mathcal{H}}(U \cup W)-e_{\mathcal{H}}(U)$ is the number of edges contained in $U \cup W$ and intersecting $W \backslash U$, whereas $e_{\mathcal{H}}(W)-e_{\mathcal{H}}(U \cap W)$ is the number of edges contained in $W$ and intersecting $W \backslash U$.

We now need some further definitions. Let $\mathcal{H}$ be a $t$-uniform hypergraph. The skeleton $\operatorname{Skel}(\mathcal{H})$ of $\mathcal{H}$ is the graph whose vertices are the essential vertices of $\mathcal{H}$, in which $x y$ is an edge if and only if $\{x, y\}$ is contained in some edge of $\mathcal{H}$. A hollow edge of $\mathcal{H}$ is an edge containing two essential and $t-2$ inessential vertices, and a full edge is an edge containing $t$ essential vertices. A $t$-polished hypergraph is a $t$-uniform hypergraph in which every edge is either hollow or full. 
The polishing of a $t$-uniform hypergraph $\mathcal{H}$ is the following procedure. For each edge $A$ with exactly $s$ essential vertices, where $s \notin\{2, t\}$, delete $A$ and add $\left(\begin{array}{c}s \\ 2\end{array}\right)$ hollow edges, each containing exactly two of these $s$ essential vertices, in such a way that all pairs are covered. Let $\operatorname{Pol}(\mathcal{H})$ denote the result of polishing $\mathcal{H}$. Then $\mathcal{H}$ is polished if and only if $\operatorname{Pol}(\mathcal{H})=\mathcal{H}$.

Lemma 12. Let $\mathcal{H}$ be a t-uniform hypergraph. Then:

(a) $\operatorname{Skel}(\operatorname{Pol}(\mathcal{H}))=\operatorname{Skel}(\mathcal{H})$.

(b) The following statements are all equivalent:

(i) $\operatorname{Skel}(\mathcal{H})$ is properly t-colourable (as a graph);

(ii) $\mathcal{H}$ is panchromatically $t$-colourable;

(iii) $\operatorname{Pol}(\mathcal{H})$ is panchromatically $t$-colourable.

(c) If $\mathcal{H}$ is well spread then so is $\operatorname{Pol}(\mathcal{H})$.

Proof. It is easy to see that (a) holds and that (i) and (ii) are equivalent in (b). The rest of (b) follows from (a).

To prove (c), assume $\mathcal{H}$ is well spread. It suffices to consider the result of replacing a single edge $A$ of $\mathcal{H}$ by $\left(\begin{array}{l}s \\ 2\end{array}\right)$ hollow edges to form a new $t$-uniform hypergraph $\mathcal{H}^{\prime}$. Suppose if possible that $W^{\prime}$ is a largest subset of $V\left(\mathcal{H}^{\prime}\right)$ for which $\operatorname{sur}_{\mathcal{H}^{\prime}}\left(W^{\prime}\right) \leqslant 0$, and suppose that $W^{\prime}$ contains exactly $r \leqslant s$ essential vertices of $A$. Then $\mathcal{E}_{\mathcal{H}^{\prime}}\left(W^{\prime}\right)$ contains exactly $\left(\begin{array}{l}r \\ 2\end{array}\right)$ of the $\left(\begin{array}{l}s \\ 2\end{array}\right)$ new hollow edges, since otherwise we could add the inessential vertices of such an edge to $W^{\prime}$, which would increase $\left|W^{\prime}\right|$ by at most $t-2$ and $e_{\mathcal{H}^{\prime}}\left(W^{\prime}\right)$ by exactly 1 and so give a larger set than $W^{\prime}$ with nonpositive surplus, by (4.1).

If $r \leqslant 1$ then $W^{\prime} \subseteq V(\mathcal{H})$ and $\mathcal{E}_{\mathcal{H}^{\prime}}\left(W^{\prime}\right) \subseteq \mathcal{E}(\mathcal{H})$, a contradiction. So we may suppose that $2 \leqslant r \leqslant s \leqslant t-1$. Let $W:=\left(W^{\prime} \cap V(\mathcal{H})\right) \cup A$. Then

$$
|W|=\left|W^{\prime}\right|-(t-2)\left(\begin{array}{l}
r \\
2
\end{array}\right)+(t-r) \text { and } e_{\mathcal{H}}(W) \geqslant e_{\mathcal{H}^{\prime}}\left(W^{\prime}\right)-\left(\begin{array}{l}
r \\
2
\end{array}\right)+1,
$$

so that

$$
\begin{aligned}
\operatorname{sur}_{\mathcal{H}}(W) & \leqslant \operatorname{sur}_{\mathcal{H}^{\prime}}\left(W^{\prime}\right)-t(t-2)\left(\begin{array}{l}
r \\
2
\end{array}\right)+t(t-r)+\left(t^{2}-2 t+2\right)\left[\left(\begin{array}{l}
r \\
2
\end{array}\right)-1\right] \\
& =\operatorname{sur}_{\mathcal{H}^{\prime}}\left(W^{\prime}\right)+t(t-r)+r(r-1)-\left(t^{2}-2 t+2\right) \\
& =\operatorname{sur}_{\mathcal{H}^{\prime}}\left(W^{\prime}\right)+(r+1-t)(r-2) \\
& \leqslant 0+0=0 .
\end{aligned}
$$

This contradiction completes the proof.

In view of Lemma 12, it suffices to prove Theorem 10 for $t$-polished hypergraphs. For the rest of the proof, therefore, we shall assume that $\mathcal{H}=$ $(V, \mathcal{E})$ is a $t$-polished counterexample to Theorem 10 such that $\operatorname{Skel}(\mathcal{H})$ has as few edges as possible and, subject to this condition, such that the sum of squares of degrees of vertices in $\mathcal{H}$ is as large as possible. Then $\mathcal{H}$ is well 
spread but $\operatorname{Skel}(\mathcal{H})$ is not $t$-colourable. In view of Lemma 12, if $\mathcal{H}^{\prime}$ is a $t$ uniform hypergraph such that $\operatorname{Skel}\left(\mathcal{H}^{\prime}\right)$ has fewer edges than $\operatorname{Skel}(\mathcal{H})$, then $\mathcal{H}^{\prime}$ is not a counterexample to Theorem 10 , regardless of whether or not $\mathcal{H}^{\prime}$ is $t$-polished. We seek a contradiction.

The next lemma is the analogue of Claim 2 in the proof of Theorem 9.

Lemma 13. Every vertex in $\operatorname{Skel}(\mathcal{H})$ has degree at least $t$.

Proof. Suppose $\operatorname{Skel}(\mathcal{H})$ contains a vertex $v$ with degree $d<t$. Construct a hypergraph $\mathcal{H}^{\prime}$ from $\mathcal{H}$ by splitting $v$ into $d$ vertices of degree 1 . It is easy to see that $\mathcal{H}^{\prime}$ satisfies (4.3) (since $\mathcal{H}$ does) and so $\mathcal{H}^{\prime}$ is well spread. Since $\operatorname{Skel}\left(\mathcal{H}^{\prime}\right)$ has fewer edges than $\operatorname{Skel}(\mathcal{H}), \mathcal{H}^{\prime}$ is not a counterexample to Theorem 10 and so $\operatorname{Skel}\left(\mathcal{H}^{\prime}\right)$ has a $t$-colouring. Now we obtain a $t$-colouring of $\operatorname{Skel}(\mathcal{H})$ by colouring $v$ differently from its neighbours, and this contradicts the choice of $\mathcal{H}$.

Lemma 14. If $A \in \mathcal{E}$ and $u \in V \backslash A$, then at least two vertices of $A$ are not adjacent to $u$.

Proof. Suppose first that every vertex of $A$ is adjacent to $u$. Then, for some $r \leqslant t$, there are $r$ edges of $\mathcal{H}$ containing $u$ that cover $A$. The union $R$ of these $r$ edges contains at most $1+r(t-1)$ vertices and at least $r+1$ edges, and so

$$
\begin{aligned}
\operatorname{sur}(R) & \leqslant t(1+r(t-1))-\left(t^{2}-2 t+2\right)(r+1)-t+2 \\
& =(r-t)(t-2) \leqslant 0
\end{aligned}
$$

a contradiction.

Now suppose that $A$ has vertices $y_{1}, \ldots, y_{t-1}, z$, where $z$ is the only vertex not adjacent to $u$. We construct a $t$-uniform hypergraph $\hat{\mathcal{H}}$ from $\mathcal{H}$ as follows: delete the edge $A$ (but not its vertices), merge $u$ and $z$ into a new vertex $(u z)$, and then, for each pair $(i, j)$ with $1 \leqslant i<j \leqslant t-1$, add a new hollow edge $B_{i, j}$ whose essential vertices are $y_{i}$ and $y_{j}$. Since $\operatorname{Skel}(\hat{\mathcal{H}})$ has fewer edges than $\operatorname{Skel}(\mathcal{H}), \hat{\mathcal{H}}$ is not a counterexample to Theorem 10. But $\operatorname{Skel}(\hat{\mathcal{H}})$ cannot have a $t$-colouring, since such a colouring would immediately yield a $t$-colouring of $\operatorname{Skel}(\mathcal{H})$ (giving $u$ and $z$ the colour of $(u z)$ ). Therefore $\hat{\mathcal{H}}$ is not well spread. Let $Z$ be a subset of $V(\hat{\mathcal{H}})$ with $\operatorname{sur}_{\hat{\mathcal{H}}}(Z) \leqslant 0$. Then $(u z) \in Z$, since $\hat{\mathcal{H}}-(u z)$ is a subhypergraph of $\operatorname{Pol}\left(\mathcal{H}^{\prime}\right)$, where $\mathcal{H}^{\prime}$ is obtained from $\mathcal{H}$ by splitting $z$ into two vertices $z^{\prime}$ (contained in $A$ only) and $z^{\prime \prime}$ (contained in all other edges that contained $z$ ), and $\operatorname{Pol}\left(\mathcal{H}^{\prime}\right)$ is well spread (since $\mathcal{H}$ is). Suppose $Z$ contains exactly $s$ vertices $y_{i}$, say $y_{1}, \ldots, y_{s}$. Then there are $r \leqslant t-1-s$ edges $A_{1}, \ldots, A_{r}$ containing $u$ that cover the remaining vertices 
$y_{s+1}, \ldots, y_{t-1}$. Let $X:=(Z \cap V) \cup\{z\} \cup \bigcup_{i=1}^{r} A_{i}$. If $Z$ contains $b$ of the edges $B_{i, j}$, then $b \leqslant\left(\begin{array}{l}s \\ 2\end{array}\right)$, and

$$
|X| \leqslant|Z|-b(t-2)+1+r(t-1) \quad \text { and } \quad e_{\mathcal{H}}(X) \geqslant e_{\hat{\mathcal{H}}}(Z)-b+1+r,
$$

so that

$$
\begin{aligned}
\operatorname{sur}_{\mathcal{H}}(X) & \leqslant \operatorname{sur}_{\hat{\mathcal{H}}}(Z)-b t(t-2)+t+r t(t-1)+\left(t^{2}-2 t+2\right)(b-1-r) \\
& =\operatorname{sur}_{\hat{\mathcal{H}}}(Z)+2 b-\left(t^{2}-3 t+2\right)+r(t-2) \\
& \leqslant 0+s(s-1)-\left(t^{2}-3 t+2\right)+(t-1-s)(t-2) \\
& =s(s-t+1) \leqslant 0 .
\end{aligned}
$$

This contradiction completes the proof.

In view of Lemma 13 , each $d$-vertex in $\mathcal{H}$ with $2 \leqslant d \leqslant t-1$ is incident with at least one full edge. A 2-vertex incident with one full edge and one hollow edge will be called a weak vertex.

We will say that a full edge $A$ is special or, more precisely, $(u, w, z)$-special, if $t$ is odd and there exist vertices $z \in A$ and $u, w \notin A$ such that

(s1) all $t-1$ vertices in $A-z$ are weak;

(s2) $u$ is adjacent to $t-2$ vertices in $A-z$ and $w$ is adjacent to the remaining vertex in $A-z$ (necessarily by hollow edges, in each case);

(s3) the degree of $u$ in $\operatorname{Skel}(\mathcal{H})$ is at least $2 t-3$;

(s4) there exists a set $Y \subseteq V$ such that $u \in Y, A \nsubseteq Y$ and $\operatorname{sur}_{\mathcal{H}}(Y)=1$.

Lemma 15. Let $A$ be a full edge and let $u$ and $w$ be distinct essential vertices in $V \backslash A$ that are adjacent to weak vertices $u_{1}, \ldots, u_{r}$ and $w_{1}, \ldots, w_{s}$ respectively in $A(r, s>0)$. Suppose that $d_{\mathcal{H}}(u) \geqslant d_{\mathcal{H}}(w)$, where $d_{\mathcal{H}}$ denotes degree in $\mathcal{H}$. Then

(i) $t$ is odd;

(ii) $s=1$;

(iii) no other essential vertex $x \in V \backslash A(x \neq u, w)$ is adjacent to weak vertices in $A$;

(iv) if $r \geqslant t-2$ then $r=t-2$ and $A$ is special.

Proof. Let $A_{i}$ (respectively, $B_{i}$ ) denote the hollow edge containing $u_{i}$ (respectively, $\left.w_{i}\right)$. Note that, by Lemma $13, u$ belongs to some edge other than $A_{1}, \ldots, A_{r}$, and similarly for $w$. Let $\mathcal{H}_{0}$ be the hypergraph obtained from $\mathcal{H}$ by deleting the edges $A_{1}, \ldots, A_{r}, B_{1}, \ldots, B_{s}$ and their inessential vertices, and adding a new hollow edge $D$ with essential vertices $u$ and $w$. Then $\operatorname{Skel}\left(\mathcal{H}_{0}\right)$ has fewer edges then $\operatorname{Skel}(\mathcal{H})$, and so $\mathcal{H}_{0}$ is not a counterexample 
to Theorem 10. But it is easy to see that a $t$-colouring of $\operatorname{Skel}\left(\mathcal{H}_{0}\right)$ could be extended to $\operatorname{Skel}(\mathcal{H})$ by giving suitable colours to $u_{1}, \ldots, u_{r}, w_{1}, \ldots, w_{s}$, and it follows that $\mathcal{H}_{0}$ cannot be well spread. Therefore $V\left(\mathcal{H}_{0}\right)$ contains a set $Y_{0}$ with nonpositive surplus. Since $\mathcal{H}$ is well spread, $D \subseteq Y_{0}$. Let $X_{0}:=Y_{0} \backslash(D \backslash\{u, w\}) \subset V(\mathcal{H})$, so that, by (4.1),

$$
\operatorname{sur}_{\mathcal{H}}\left(X_{0}\right)=\operatorname{sur}_{\mathcal{H}_{0}}\left(Y_{0}\right)-t(t-2)+\left(t^{2}-2 t+2\right) \leqslant 2 .
$$

Note that $X_{0}$ contains $u$ and $w$ but no inessential vertices of any edges $A_{1}, \ldots, A_{r}, B_{1}, \ldots, B_{s}$. If $u_{i} \in X_{0}$ for some $i$ then

$$
\operatorname{sur}_{\mathcal{H}}\left(X_{0} \cup A_{i}\right)=\operatorname{sur}_{\mathcal{H}}\left(X_{0}\right)+t(t-2)-\left(t^{2}-2 t+2\right) \leqslant 0,
$$

a contradiction. So $X_{0}$ contains no vertex $u_{i}$ and, similarly, no vertex $w_{i}$.

Let $A_{r+1}^{\prime}:=B_{s}-w+u$ and let $\mathcal{H}_{1}$ be the hypergraph with $V\left(\mathcal{H}_{1}\right)=V$ and $\mathcal{E}\left(\mathcal{H}_{1}\right)=\mathcal{E}-B_{s}+A_{r+1}^{\prime}$. Intuitively, $\mathcal{H}_{1}$ is formed from $\mathcal{H}$ by detaching $B_{s}$ from $w$ and reattaching it at $u$. $\operatorname{Skel}\left(\mathcal{H}_{1}\right)$ has fewer edges than $\operatorname{Skel}(\mathcal{H})$ if $w$ is inessential in $\mathcal{H}_{1}$ and the same number otherwise, and $\mathcal{H}_{1}$ has larger sum of squares of degrees than $\mathcal{H}$. Thus $\mathcal{H}_{1}$ is not a counterexample to Theorem 10. So if $\mathcal{H}_{1}$ is well spread then $\operatorname{Skel}\left(\mathcal{H}_{1}\right)$ has a $t$-colouring $\phi$. If $\phi(u)=\phi(w)$ then $\phi$ is a $t$-colouring of $\operatorname{Skel}(\mathcal{H})$, otherwise it gives a $t$ colouring of $\operatorname{Skel}\left(\mathcal{H}_{0}\right)$ and can be extended to $\operatorname{Skel}(\mathcal{H})$ as in the previous paragraph. This contradiction shows that $\mathcal{H}_{1}$ cannot be well spread. Choose a set $Y_{1} \subseteq V\left(\mathcal{H}_{1}\right)$ with nonpositive surplus. Since $\mathcal{H}$ is well spread, $A_{r+1}^{\prime} \subset Y_{1}$. Let $X_{1}:=Y_{1} \backslash\left(A_{r+1}^{\prime} \backslash\left\{u, w_{s}\right\}\right)$, so that, by (4.1),

$$
\operatorname{sur}_{\mathcal{H}}\left(X_{1}\right)=\operatorname{sur}_{\mathcal{H}_{1}}\left(Y_{1}\right)-t(t-2)+\left(t^{2}-2 t+2\right) \leqslant 2 .
$$

If $A \not \subset X_{1}$ then we can delete $w_{s}$ from $X_{1}$ without deleting any edges from $\mathcal{E}_{\mathcal{H}}\left(X_{1}\right)$, and so

$$
\operatorname{sur}_{\mathcal{H}}\left(X_{1}-w_{s}\right)=\operatorname{sur}_{\mathcal{H}}\left(X_{1}\right)-t<0,
$$

a contradiction; hence $A \subset X_{1}$. If $w \in X_{1}$ then

$$
\operatorname{sur}_{\mathcal{H}}\left(X_{1} \cup B_{s}\right)=\operatorname{sur}_{\mathcal{H}}\left(X_{1}\right)+t(t-2)-\left(t^{2}-2 t+2\right) \leqslant 0,
$$

a contradiction; so $w \notin X_{1}$. Now the same argument as in (4.4) shows that $X_{1}$ contains no inessential vertex of any set $B_{i}$.

Since $u \in X_{0} \cap X_{1}$, (4.2) gives $\operatorname{sur}_{\mathcal{H}}\left(X_{0} \cap X_{1}\right) \geqslant 1$, and Lemma 11 implies

$$
\begin{aligned}
\operatorname{sur}_{\mathcal{H}}\left(X_{0} \cup X_{1}\right) & \leqslant \operatorname{sur}_{\mathcal{H}}\left(X_{0}\right)+\operatorname{sur}_{\mathcal{H}}\left(X_{1}\right)-\operatorname{sur}_{\mathcal{H}}\left(X_{0} \cap X_{1}\right) \\
& \leqslant 2+2-1=3 .
\end{aligned}
$$


Let $X:=X_{0} \cup X_{1} \cup \bigcup_{i=1}^{s} B_{i}$. By the final sentence of the previous paragraph, (4.6) $\operatorname{sur}_{\mathcal{H}}(X)=\operatorname{sur}_{\mathcal{H}}\left(X_{0} \cup X_{1}\right)+t(t-2) s-\left(t^{2}-2 t+2\right) s \leqslant 3-2 s$.

By (4.2), (4.5) and (4.6), $s=1, \operatorname{sur}_{\mathcal{H}}(X)=\operatorname{sur}_{\mathcal{H}}\left(X_{0} \cap X_{1}\right)=1$, and $\operatorname{sur}_{\mathcal{H}}\left(X_{0}\right)=$ $\operatorname{sur}_{\mathcal{H}}\left(X_{1}\right)=2$. But by (4.1), $\operatorname{sur}_{\mathcal{H}}(X)$ is even if $t$ is even, and so $t$ is odd. Hence (i) and (ii) hold.

It is convenient next to prove (iv). If $r \geqslant t-2$, then $r=t-2$ by Lemma 14 . Let $z$ be the vertex in $A \backslash\left\{u_{1}, \ldots, u_{r}, w_{1}\right\}$, and let $Y:=X_{0} \cap X_{1}$. Then $u \in Y$, and (s1), (s2) and (s4) (in the definition of a special vertex) all hold. If (s3) does not hold, then $u$ has degree at most $t-2$ in $\operatorname{Skel}\left(\mathcal{H}_{2}\right)$, where $\mathcal{H}_{2}$ is obtained from $\mathcal{H}$ by deleting $A_{1}, \ldots, A_{r}$ and their inessential vertices. By the minimality of $\mathcal{H}, \operatorname{Skel}\left(\mathcal{H}_{2}\right)$ has a $t$-colouring, in which $u$ can be recoloured if necessary with a different colour from $w$. This colouring can easily be extended to $\operatorname{Skel}(\mathcal{H})$, and this contradiction proves (s3) and hence (iv).

Finally, we must prove (iii). Suppose there is a vertex $x \in V \backslash A$, different from $u$ and $w$, that is adjacent to a weak vertex $x_{1} \in A$. W.l.o.g. we may assume that $d_{\mathcal{H}}(u) \geqslant d_{\mathcal{H}}(w) \geqslant d_{\mathcal{H}}(x)$. Let $C_{1}$ be the hollow edge containing $x$ and $x_{1}$. Recall that the sets $X_{0}$ and $X_{1}$ satisfy

$A_{1} \cap X_{0}=\{u\}, \quad B_{1} \cap X_{0}=\{w\}, u \in X_{1}, \quad A \subset X_{1}$ and $B_{1} \cap X_{1}=\left\{w_{1}\right\}$.

By the same argument, there exist subsets $U_{0}, U_{1}, W_{0}, W_{1}$ of $V$, all with surplus 2, such that

$A_{1} \cap W_{0}=\{u\}, \quad C_{1} \cap W_{0}=\{x\}, u \in W_{1}, \quad A \subset W_{1}$ and $C_{1} \cap W_{1}=\left\{x_{1}\right\}$, $B_{1} \cap U_{0}=\{w\}, \quad C_{1} \cap U_{0}=\{x\}, w \in U_{1}, \quad A \subset U_{1}$ and $C_{1} \cap U_{1}=\left\{x_{1}\right\}$. Possibly, two sets among $X_{0}, W_{0}, U_{0}$ or among $X_{1}, W_{1}, U_{1}$ may coincide.

If $C_{1} \subset X_{0}$, let $X_{0}^{\prime}:=X_{0} \backslash\left(C_{1}-x\right)$. Then

$$
\operatorname{sur}\left(X_{0}^{\prime}\right)=\operatorname{sur}\left(X_{0}\right)-t(t-1)+\left(t^{2}-2 t+2\right)<\operatorname{sur}\left(X_{0}\right),
$$

and using $X_{0}^{\prime}$ instead of $X_{0}$ in the definition of $X$ would give $\operatorname{sur}(X) \leqslant$ $2-2 s=0$ in place of (4.6), a contradiction. Therefore $C_{1} \not \subset X_{0}$. Now the same argument as in (4.4) shows that $X_{0}$ contains no vertex of $C_{1}$ except possibly for $x$. By an exactly similar argument, $W_{0}$ contains no vertex of $B_{1}$ except possibly for $w$. Thus

$$
\begin{gathered}
\text { none of } U_{0}, W_{0} \text { and } X_{0} \text { contain any vertices of } B_{1} \text { or } C_{1} \\
\text { except for } w \text { and } x .
\end{gathered}
$$

Note that $W_{1} \cap\left(W_{0} \cup X_{0}\right) \neq \emptyset$ since $u$ is in all three sets. So, by (4.2), two applications of Lemma 11 give

$$
\operatorname{sur}\left(W_{0} \cup X_{0}\right) \leqslant \operatorname{sur}\left(W_{0}\right)+\operatorname{sur}\left(X_{0}\right)-\operatorname{sur}\left(W_{0} \cap X_{0}\right) \leqslant 2+2-1=3
$$


and

$$
\begin{gathered}
\operatorname{sur}\left(W_{1} \cup W_{0} \cup X_{0}\right) \leqslant \operatorname{sur}\left(W_{1}\right)+\operatorname{sur}\left(W_{0} \cup X_{0}\right) \\
\quad-\operatorname{sur}\left(W_{1} \cap\left(W_{0} \cup X_{0}\right)\right) \leqslant 2+3-1=4 .
\end{gathered}
$$

If $w \notin W_{1}$ then, by the argument of (4.4), $W_{1}$ contains no inessential vertices of $B_{1}$, and we already know that it contains no inessential vertices of $C_{1}$. Therefore, by (4.7) and since $A \subset W_{1}$,

$$
\begin{gathered}
\operatorname{sur}\left(W_{1} \cup W_{0} \cup X_{0} \cup B_{1} \cup C_{1}\right) \\
=\operatorname{sur}\left(W_{1} \cup W_{0} \cup X_{0}\right)+2 t(t-2)-2\left(t^{2}-2 t+2\right) \leqslant 4-4=0 .
\end{gathered}
$$

This contradiction shows that $w \in W_{1}$. An exactly similar argument shows that $x \in X_{1}$. Thus

$$
w \in W_{1} \cap U_{0} \quad \text { and } \quad x \in X_{1} \cap U_{0} .
$$

Let $Z:=W_{1} \cap X_{1}$. Note that $u \in Z$ and $Z$ contains no vertices of $B_{1}$ or $C_{1}$ except for $w_{1}$ and $x_{1}$. So if $\operatorname{sur}(Z) \leqslant 2$ then we can argue with $Z$ as we did above with $W_{1}$ (starting in the line after (4.7)) to get a contradiction, since $w \notin Z$. On the other hand,

$$
\operatorname{sur}(Z) \leqslant \operatorname{sur}\left(W_{1}\right)+\operatorname{sur}\left(X_{1}\right)-\operatorname{sur}\left(W_{1} \cup X_{1}\right) \leqslant 2+2-1=3 .
$$

So $\operatorname{sur}(Z)=3$. If $Z \cap U_{0} \neq \emptyset$ then

$$
\operatorname{sur}\left(Z \cup U_{0}\right) \leqslant \operatorname{sur}(Z)+\operatorname{sur}\left(U_{0}\right)-\operatorname{sur}\left(Z \cap U_{0}\right) \leqslant 3+2-1=4
$$

and, by (4.7),

$\operatorname{sur}\left(Z \cup U_{0} \cup B_{1} \cup C_{1}\right) \leqslant \operatorname{sur}\left(Z \cup U_{0}\right)+2 t(t-2)-2\left(t^{2}-2 t+2\right) \leqslant 4-4=0$, a contradiction. So $Z \cap U_{0}=\emptyset$. Let $Y:=\left(W_{1} \cup X_{1}\right) \cap U_{0}$. Then $|Y|=\left|W_{1} \cap U_{0}\right|+$ $\left|X_{1} \cap U_{0}\right|$ and $e(Y)=e\left(W_{1} \cap U_{0}\right)+e\left(X_{1} \cap U_{0}\right)$ and so, by (4.1) and (4.8),

$$
\begin{gathered}
\operatorname{sur}(Y)=\operatorname{sur}\left(W_{1} \cap U_{0}\right)+\operatorname{sur}\left(X_{1} \cap U_{0}\right)+t-2 \geqslant 1+1+t-2=t, \\
\operatorname{sur}\left(W_{1} \cup X_{1}\right) \leqslant \operatorname{sur}\left(W_{1}\right)+\operatorname{sur}\left(X_{1}\right)-\operatorname{sur}(Z)=2+2-3=1,
\end{gathered}
$$

and

$$
\operatorname{sur}\left(W_{1} \cup X_{1} \cup U_{0}\right) \leqslant \operatorname{sur}\left(W_{1} \cup X_{1}\right)+\operatorname{sur}\left(U_{0}\right)-\operatorname{sur}(Y) \leqslant 1+2-t \leqslant 0,
$$

a contradiction. This completes the proof of Lemma 15.

Suppose that some edge $A$ contains three or more weak vertices. By Lemmas 14 and 15, $t \geqslant 5$ and there is a unique vertex $u \in V \backslash A$ that is 
adjacent to at least two weak vertices of $A$; we define $u(A):=u$. We will say that a vertex $u=u(A)$ is $A$-special if the edge $A$ is $(u, w, z)$-special for some $w$ and $z$, and that $u$ is very special if it is $A$-special for some $A$ and $d_{\mathcal{H}}(u)=t-1$; in this last case, by (s3), $u$ is incident with exactly one full edge $B \neq A$ and exactly $t-2$ hollow edges intersecting $A$.

For a positive integer $k$, we will say that a vertex $v$ is $k$-full if it belongs to at least two full edges or to one full edge and at least $k$ hollow edges. Much of the rest of the proof of Theorem 10 is contained in the following long lemma, the hypotheses of which imply $t \geqslant 5$.

Lemma 16. Let $A$ be a full edge containing exactly $m \geqslant 3$ weak vertices. Then one of the following alternatives holds:

(i) there are two $m$-full vertices in $A$ that are not very special;

(ii) for some $j(3 \leqslant j \leqslant t-m)$ there are $j(t-j+1)$-full vertices in $A$ that are not very special;

(iii) $A$ is special and the nonweak vertex in $A$ is $(t-1)$-full.

Proof. Suppose that the result is false. Suppose that $A$ contains weak vertices $u_{1}, \ldots, u_{m}$ and nonweak vertices $v_{1} \ldots, v_{t-m}$. Then $A=A_{\mathrm{w}} \cup A_{\mathrm{n}}$, where $A_{\mathrm{w}}:=\left\{u_{1}, \ldots, u_{m}\right\}$ and $A_{\mathrm{n}}:=\left\{v_{1} \ldots, v_{t-m}\right\}$. Suppose $u=u(A)$ is adjacent to exactly $r$ weak vertices $u_{1}, \ldots, u_{r}$. By Lemmas 14 and $15,2 \leqslant r \leqslant t-2, r=m$ or $m-1$ (so $m \leqslant t-1$ ), and $A$ is special if and only if $m=t-1$, when $r=t-2$. If $r=m-1$ let $w$ be the essential vertex in $V \backslash A$ that is adjacent to $u_{m}$. If $m \leqslant t-2$ then it is possible that some vertices $v_{i} \in A_{\mathrm{n}}$ are adjacent to $u$ (but not if $v_{i}$ is very special). If $v_{i}$ is very special, we will assume that $v_{i}$ is $D_{i}$-special, where $D_{i}$ is $\left(v_{i}, w_{i}, z_{i}\right)$-special.

Let $\mathcal{F}:=\mathcal{H}-A$; that is, $\mathcal{F}$ is obtained from $\mathcal{H}$ by deleting the edge $A$ (but not its vertices). Let $F$ be the graph whose vertices are the essential vertices of $\mathcal{F}$ together with all of $v_{1}, \ldots, v_{t-m}$ (which may or may not be essential in $\mathcal{F}$ ) and also $u_{m}$ if $r=m-1$, and in which $x y$ is an edge if and only if $\{x, y\}$ is contained in some edge of $\mathcal{F}$; thus $\operatorname{Skel}(\mathcal{F}) \subseteq F$. Note that $F$ includes every vertex of $A$ that is not adjacent to $u$. Also, for $k<t$, a vertex $v_{i} \in A_{\mathrm{n}}$ is necessarily $k$-full if it has degree at least $k$ in $F$. The proof proceeds by a sequence of seven claims.

Claim 1. There cannot exist a $t$-colouring $\phi$ of $F$ in which $\phi(y)=\phi(u)$ for some $y \in A$.

Proof. Suppose there exists such a $\phi$. Clearly $y$ is not adjacent to $u$, and so either $y=u_{m}$ and $r=m-1$, or else $y=v_{i} \in A_{\mathrm{n}}$ for some $i$. If there is no $i$ such that $\phi\left(v_{i}\right)=\phi(u)$, then set $i:=0$. Uncolour all vertices of $A \cap V(F)$. We shall show that, by recolouring $v_{1}, \ldots, v_{t-m}$, we can ensure that they all 
have different colours and, if $i \neq 0$, then one of them has the same colour as $u$; it is then easy to colour $u_{1}, \ldots, u_{m}$ so as to give a $t$-colouring of $\operatorname{Skel}(\mathcal{H})$, a contradiction.

If $m=t-1$ then $v_{1}$ can keep its old colour, since if $\phi\left(v_{1}\right) \neq \phi(u)$ then $\phi\left(u_{m}\right)=\phi(u)$. Hence we may suppose that $m \leqslant t-2$, so that $A$ is not special. We may suppose that the vertices $v_{1}, \ldots, v_{t-m}$ are labelled so that those that are not very special come first, in nonincreasing order of degree, followed by those that are very special, in any order. It then follows that, for each $j$ $(2 \leqslant j \leqslant t-m)$, either $v_{j}$ is very special or $d_{F}\left(v_{j}\right) \leqslant t-j$,

where $d_{F}$ denotes degree in $F$; for, if (4.9) fails, then $v_{1}, \ldots, v_{j}$ are not very special and all have degree at least $t-j+1$ in $F$, which means that they are $(t-j+1)$-full (hence, if $j=2$, they are more than $m$-full). We now recolour $v_{1}, \ldots, v_{t-m}$ in order, ensuring that they all have different colours and that at least one has colour $\phi(u)$. Give $v_{1}$ colour $\phi\left(v_{1}\right)$; then, for $j=2, \ldots, t-m$ in turn, do the following:

(a) if $j=i$ : if none of $v_{1}, \ldots, v_{i-1}$ has been given colour $\phi(u)$, then give $v_{i}$ colour $\phi\left(v_{i}\right)=\phi(u)$; otherwise, colour $v_{i}$ exactly as in (b) or (c) below (that is, like $v_{j}$ where $j \neq i$ );

(b) if $j \neq i$ and $v_{j}$ is not very special, then $v_{j}$ is adjacent to at most $t-j$ vertices of $F$ by (4.9), so colour it differently from all of these and from $v_{1}, \ldots, v_{j-1}$;

(c) if $j \neq i$ and $v_{j}$ is very special, then colour $v_{j}$ differently from $v_{1}, \ldots, v_{j-1}$ and from $w_{j}$, and recolour the weak vertices of $D_{j}$ if necessary.

It is now easy to obtain a $t$-colouring of $\operatorname{Skel}(\mathcal{H})$, a contradiction.

Claim 2. Every vertex $v_{i} \in A_{\mathrm{n}}$ that is adjacent to $u$ is $t$-full.

Proof. Suppose that $v_{i}$ belongs only to $A$ and to some $q \leqslant t-1$ hollow edges, one of which contains $u$. Let $B$ be a hollow edge containing $v_{i}$ but not $u$, which must exist since $v_{i}$ is not weak. (It is clear that our minimal counterexample $\mathcal{H}$ cannot contain two hollow edges joining the same pair of essential vertices.) By the minimality of $\mathcal{H}$, there is a $t$-colouring of $\operatorname{Skel}(\mathcal{H}-B)$, in which some vertex $y \neq v_{i}$ of $A$ has the same colour as $u$. Recolouring $v_{i}$ differently from its $q$ essential neighbours outside $A$, we obtain a $t$-colouring of $F$ violating Claim 1. This contradiction proves Claim 2.

Claim 3. For each $y \in A$ not adjacent to $u$, there exists a set $U_{y} \subseteq V$ containing $u$ and $y$ such that $\operatorname{sur}_{\mathcal{F}}\left(U_{y}\right) \leqslant t$. 
Proof. Let $\mathcal{F}_{y}$ be obtained from $\mathcal{F}$ by merging $u$ and $y$ into a new vertex (uy). $\mathcal{F}_{y}$ has smaller skeleton than $\mathcal{H}$, and so it is not a counterexample to Theorem 10. But a panchromatic $t$-colouring of $\mathcal{F}_{y}$ would give a $t$-colouring of $F$ violating Claim 1. Thus $\mathcal{F}_{y}$ is not well spread; that is, there exists a set $U_{y}^{\prime} \subseteq V\left(\mathcal{F}_{y}\right)$ with $\operatorname{sur}_{\mathcal{F}_{y}}\left(U_{y}^{\prime}\right) \leqslant 0$. Since $\mathcal{H}$ is well spread, $(u y) \in U_{y}^{\prime}$. Then $U_{y}:=U_{y}^{\prime}-(u y)+u+y$ satisfies the Claim.

Claim 4. For each vertex $v_{i} \in A_{\mathrm{n}}$ that is not very special and not adjacent to $u$ and for which $d_{F}\left(v_{i}\right) \leqslant r$, there exists a set $Z_{i} \subseteq V$ containing $u$ and $v_{i}$ such that $\operatorname{sur}_{\mathcal{F}}\left(Z_{i}\right) \leqslant t+1-d_{F}\left(v_{i}\right)$.

Proof. Let $q:=d_{F}\left(v_{i}\right)$, so that $2 \leqslant q \leqslant r$ (since $v_{i}$ is not weak). Let $B_{1}, \ldots, B_{q}$ be the hollow edges containing $v_{i}$ and let $b_{1}, \ldots, b_{q}$ be the other essential vertices in these edges. For $j=1, \ldots, q$, let $B_{j}^{\prime}:=B_{j}-v_{i}+u$ and let $\mathcal{H}_{j}$ be the hypergraph with $V\left(\mathcal{H}_{j}\right)=V$ and $\mathcal{E}\left(\mathcal{H}_{j}\right)=\mathcal{E}-B_{j}+B_{j}^{\prime}$. Then $\operatorname{Skel}(\mathcal{H})$ and $\operatorname{Skel}\left(\mathcal{H}_{j}\right)$ have the same number of edges, but the sum of squares of degrees in $\mathcal{H}_{j}$ is greater, since $q \leqslant r$. (Note that, by Lemma 13, there must be at least one edge in $\mathcal{H}$ containing $u$, in addition to the $r$ hollow edges containing $u$ and $u_{1}, \ldots, u_{r}$.) Thus Theorem 10 holds for $\mathcal{H}_{j}$.

Suppose that $\mathcal{H}_{j}$ has a panchromatic t-colouring $\phi$. If $\phi\left(v_{i}\right) \neq \phi\left(b_{j}\right)$, then $\phi$ gives a $t$-colouring of $\operatorname{Skel}(\mathcal{H})$. If $\phi\left(v_{i}\right)=\phi\left(b_{j}\right)(\neq \phi(u))$, then some $y \in A-v_{i}$ has the same colour as $u$, and recolouring $v_{i}$ differently from $b_{1}, \ldots, b_{q}$ gives a $t$-colouring of $F$ violating Claim 1 . Thus $\mathcal{H}_{j}$ has no panchromatic $t$-colouring and so is not well spread; that is, there exists $Y_{j}^{\prime} \subseteq V\left(\mathcal{H}_{j}\right)$ with nonpositive surplus. Since $\mathcal{H}$ is well spread, $B_{j}^{\prime} \subseteq Y_{j}^{\prime}$. If $v_{i} \in Y_{j}^{\prime}$ then $\operatorname{sur}_{\mathcal{H}}\left(Y_{j}^{\prime}\right)=\operatorname{sur}_{\mathcal{H}_{j}}\left(Y_{j}^{\prime}\right) \leqslant 0$, a contradiction; so $v_{i} \notin Y_{j}^{\prime}$. Let $Y_{j}:=Y_{j}^{\prime} \backslash\left(B_{j}^{\prime} \backslash\left\{u, b_{j}\right\}\right)$. Then

$$
\operatorname{sur}_{\mathcal{F}}\left(Y_{j}\right) \leqslant \operatorname{sur}_{\mathcal{H}_{j}}\left(Y_{j}^{\prime}\right)-t(t-2)+\left(t^{2}-2 t+2\right) \leqslant 2 .
$$

Since $u \in Y_{1} \cap \ldots \cap Y_{q}$, we can make $q-1$ applications of Lemma 11 to get

$$
\begin{aligned}
\operatorname{sur}_{\mathcal{F}}\left(Y_{1} \cup \ldots \cup Y_{q}\right) & \leqslant \operatorname{sur}_{\mathcal{F}}\left(Y_{1} \cup \ldots \cup Y_{q-1}\right)+\operatorname{sur}_{\mathcal{F}}\left(Y_{1}\right)-1 \\
& \leqslant \sum_{j=1}^{q} \operatorname{sur}_{\mathcal{F}}\left(Y_{j}\right)-(q-1) \leqslant q+1 .
\end{aligned}
$$

Let $Z_{i}:=\bigcup_{j=1}^{q}\left(Y_{j} \cup B_{j}\right)$. Since $\left\{b_{1}, \ldots, b_{q}\right\} \subset Y_{1} \cup \ldots \cup Y_{q}$ and $v_{i} \notin Y_{1} \cup \ldots \cup Y_{q}$, therefore

$$
\left|Z_{i}\right| \leqslant\left|Y_{1} \cup \ldots \cup Y_{q}\right|+q(t-2)+1 \text { and } e_{\mathcal{F}}\left(Z_{i}\right) \geqslant e_{\mathcal{F}}\left(Y_{1} \cup \ldots \cup Y_{q}\right)+q \text {, }
$$

so that

$$
\begin{gathered}
\operatorname{sur}_{\mathcal{F}}\left(Z_{i}\right) \leqslant \operatorname{sur}_{\mathcal{F}}\left(Y_{1} \cup \ldots \cup Y_{q}\right)+q t(t-2)+t-q\left(t^{2}-2 t+2\right) \\
\leqslant q+1+t-2 q=t+1-q,
\end{gathered}
$$

as claimed. 
Claim 5. For each vertex $v_{i} \in A_{n}$ that is very special and not adjacent to $u$, there exists a set $Z_{i} \subseteq V$ containing $u$ and $v_{i}$ such that $\operatorname{sur}_{\mathcal{F}}\left(Z_{i}\right) \leqslant t-2$.

Proof. Let $B_{1}, \ldots, B_{t-2}$ be the hollow edges containing $v_{i}$ and let $b_{1}, \ldots, b_{t-2}$ be the other essential vertices in these edges. Recall that $b_{1}, \ldots, b_{t-2}$ are weak vertices contained in $D_{i}$. Let $h:=t-r-1$. For $j=1, \ldots, h$, let $B_{j}^{\prime}:=B_{j}-v_{i}+u$. Let $\mathcal{H}^{\prime}$ be the hypergraph with $V\left(\mathcal{H}^{\prime}\right)=V$ and $\mathcal{E}\left(\mathcal{H}^{\prime}\right)=\mathcal{E}-B_{1}-\ldots-B_{h}+$ $B_{1}^{\prime}+\ldots+B_{h}^{\prime}$. Skel $\left(\mathcal{H}^{\prime}\right)$ and $\operatorname{Skel}(\mathcal{H})$ have the same number of edges, but the sum of squares of degrees of vertices is larger in $\mathcal{H}^{\prime}$ than in $\mathcal{H}$; hence $\mathcal{H}^{\prime}$ is not a counterexample to Theorem 10 .

Suppose that $\mathcal{H}^{\prime}$ has a panchromatic $t$-colouring $\phi$. If $\phi(u)=\phi\left(v_{i}\right)$, then $\phi$ is a panchromatic $t$-colouring of $\mathcal{H}$. If $\phi(u) \neq \phi\left(v_{i}\right)$, then some $y \in A-v_{i}$ has the same colour as $u$, and after recolouring $v_{i}$ differently from $w_{i}$ and the nonweak vertices in $A-v_{i}$, we can then recolour the weak vertices in $A \cup D_{i}$ so as to get a $t$-colouring of $\operatorname{Skel}(\mathcal{H})$. In both cases we have a contradiction. Thus $\mathcal{H}^{\prime}$ has no panchromatic $t$-colouring and so is not well spread; that is, there exists $Y^{\prime} \subseteq V\left(\mathcal{H}^{\prime}\right)$ with nonpositive surplus. Choose $Y^{\prime}$ to have minimum possible surplus. Since $\mathcal{H}$ is well spread, some $B_{j}^{\prime}(1 \leqslant j \leqslant h)$ is contained in $Y^{\prime}$ and $v_{i} \notin Y^{\prime}$. By the minimality of $\operatorname{sur}_{\mathcal{H}^{\prime}}\left(Y^{\prime}\right), D_{i} \subset Y^{\prime}$ (otherwise we could reduce $\operatorname{sur}_{\mathcal{H}^{\prime}}\left(Y^{\prime}\right)$ by deleting all vertices of $B_{j}^{\prime}-u$ from $\left.Y^{\prime}\right)$. Let $Z_{i}:=Y^{\prime} \cup \bigcup_{s=h+1}^{t-2} B_{s}$. Then, since $t-2-h=r-1$,

$$
\left|Z_{i}\right| \leqslant\left|Y^{\prime}\right|+1+(t-2)(r-1) \text { and } e_{\mathcal{F}}\left(Z_{i}\right) \geqslant e_{\mathcal{H}^{\prime}}\left(Y^{\prime}\right)+(r-1),
$$

so that

$$
\begin{aligned}
\operatorname{sur}_{\mathcal{F}}\left(Z_{i}\right) & \leqslant \operatorname{sur}_{\mathcal{H}^{\prime}}\left(Y^{\prime}\right)+t+t(t-2)(r-1)-\left(t^{2}-2 t+2\right)(r-1) \\
& \leqslant 0+t-2(r-1) \leqslant t-2
\end{aligned}
$$

as required.

We now introduce an alternative labelling of the vertices of $A$. Let $x_{1}, \ldots, x_{t-k}$ be those that are adjacent to $u$ and let $y_{1}, \ldots, y_{k}$ be those that are not adjacent to $u$. The former include $u_{1}, \ldots, u_{r}$, and the latter include $u_{m}$ if $r=m-1$. Note that $k \geqslant 2$ by Lemma 14 , and so $2 \leqslant k \leqslant t-r$. For each $j(1 \leqslant j \leqslant k)$, choose $W_{j}$ containing $u$ and $y_{j}$ so that $\operatorname{sur}_{\mathcal{F}}\left(W_{j}\right)$ is as small as possible, and let $s_{j}:=\operatorname{sur}_{\mathcal{F}}\left(W_{j}\right)$.

Claim 6. There exists $Z \subset V$ such that $A \subset Z$ and $\operatorname{sur}_{\mathcal{H}}(Z) \leqslant$ $\sum_{j=1}^{k}\left(s_{j}-t+1\right)-1$. 
Proof. Let $Y:=\bigcup_{j=1}^{k} W_{j}$. Since $u \in W_{j}$ for each $j$, we can make $k-1$ applications of Lemma 11 exactly as in (4.10) to deduce that

$$
\operatorname{sur}_{\mathcal{F}}(Y) \leqslant \sum_{j=1}^{k} s_{j}-(k-1) .
$$

Since $A \backslash Y \subseteq\left\{x_{1}, \ldots, x_{t-k}\right\}$, for some $h \leqslant t-k$ there exist edges $A_{1}, \ldots, A_{h}$ such that $u \in A_{i} \not Y$ for each $i$ and, if we define $Z:=Y \cup \bigcup_{i=1}^{t-k} A_{i}$, then $A \subset Z$. Thus

$$
|Z| \leqslant|Y|+(t-1) h \quad \text { and } \quad e_{\mathcal{F}}(Z) \geqslant e_{\mathcal{F}}(Y)+h,
$$

and so

$$
\begin{aligned}
\operatorname{sur}_{\mathcal{F}}(Z) & \leqslant \operatorname{sur}_{\mathcal{F}}(Y)+t(t-1) h-\left(t^{2}-2 t+2\right) h \\
& =\operatorname{sur}_{\mathcal{F}}(Y)+(t-2) h \\
& \leqslant \operatorname{sur}_{\mathcal{F}}(Y)+(t-2)(t-k) .
\end{aligned}
$$

Since $e_{\mathcal{H}}(Z)=e_{\mathcal{F}}(Z)+1,(4.11)$ and (4.12) give

$$
\begin{aligned}
\operatorname{sur}_{\mathcal{H}}(Z) & \leqslant \sum_{j=1}^{k} s_{j}-(k-1)+(t-2)(t-k)-\left(t^{2}-2 t+2\right) \\
& =\sum_{j=1}^{k} s_{j}-k t+k-1,
\end{aligned}
$$

as required.

By Claim $3, s_{j} \leqslant t$ for all $j$. There is at most one value of $j$ for which $y_{j}$ is weak, which occurs if $y_{j}=u_{m}$ and $r=m-1$. If $y_{j}$ is not weak, then by Claims 4 and $5, s_{j} \leqslant t-1$ unless $y_{j}$ is not very special and $d_{F}\left(y_{j}\right)>r$, which means that $y_{j}$ is $(r+1)$-full and hence $m$-full. Since alternative (i) does not hold in the statement of the Lemma, there is at most one vertex $y_{j}$ of this last type. Thus in order for Claim 6 not to lead to the contradiction $\operatorname{sur}_{\mathcal{H}}(Z) \leqslant 0$, the following must all hold:

(a) $s_{j} \geqslant t-1$ for each $j$;

(b) $r=m-1$ (we will assume $y_{1}=u_{m}$ ) and $s_{1}=t$;

(c) exactly one $y_{j}$, say $y_{k}$, is $m$-full, and $y_{k}$ is not very special and $s_{k}=t$;

(d) (by (a) and Claims 4 and 5) for $2 \leqslant j \leqslant k-1, y_{j}$ is not very special and $d_{\mathcal{F}}\left(y_{j}\right)=d_{F}\left(y_{j}\right)=2$;

(e) (by (b) and Lemma 15) $t$ is odd. 
If $m=t-1$, then by (b), (c) and Lemma 15, alternative (iii) holds in the statement of the Lemma. So we may suppose that $m \leqslant t-2$. Since a nonweak vertex in $A$ adjacent to $u$ is clearly not very special, and is $t$-full and hence $m$-full by Claim 2, and since alternative (i) does not hold, by (c) there are no such vertices; hence $k=t-m+1 \geqslant 3$. By (d) there is a vertex $y_{2}$ with degree 2 in $F$. We will prove that there exists a set $W_{2}^{\prime} \subseteq V$ containing $u, y_{1}$ and $y_{2}$ such that $\operatorname{sur}_{\mathcal{F}}\left(W_{2}^{\prime}\right) \leqslant 2 t-3=s_{1}+s_{2}-2$. Then the argument of Claim 6

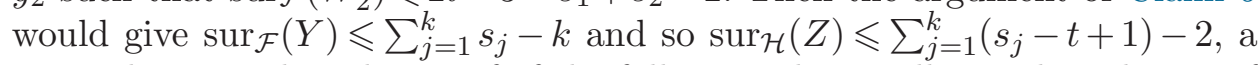
contradiction. Thus the proof of the following claim will complete the proof of Lemma 16.

Claim 7. There exists $W \subset V$ containing $u, y_{1}$ and $y_{2}$ such that $\operatorname{sur}_{\mathcal{F}}(W) \leqslant$ $2 t-3$.

Proof. Let $B_{0}$ be the hollow edge containing $y_{1}$, with essential vertices $w$ and $y_{1}=u_{m}$. Let $B_{1}$ and $B_{2}$ be the hollow edges containing $y_{2}$, and let $b_{1}$ and $b_{2}$ be the other essential vertices in these edges. It suffices to prove the existence of a set $X$ such that

$$
\left\{u, w, b_{1}, b_{2}\right\} \subset X \quad \text { and } \quad \operatorname{sur}_{\mathcal{F}}(X) \leqslant 3 .
$$

For, let

$$
X^{\prime}:=\left\{\begin{array}{ll}
X & \text { if } y_{1} \in X, \\
X \cup B_{0} & \text { if } y_{1} \notin X,
\end{array} \quad \text { and } \quad W:= \begin{cases}X^{\prime} & \text { if } y_{2} \in X, \\
X^{\prime} \cup B_{1} \cup B_{2} & \text { if } y_{2} \notin X .\end{cases}\right.
$$

Then $W$ contains $u, y_{1}$ and $y_{2}$, and since $t>4$ by the hypotheses of Lemma 16,

$$
\begin{gathered}
\operatorname{sur}_{\mathcal{F}}\left(X^{\prime}\right)-\operatorname{sur}_{\mathcal{F}}(X) \leqslant t(t-1)-\left(t^{2}-2 t+2\right)=t-2, \\
\operatorname{sur}_{\mathcal{F}}(W)-\operatorname{sur}_{\mathcal{F}}\left(X^{\prime}\right) \leqslant t(2 t-3)-2\left(t^{2}-2 t+2\right)=t-4,
\end{gathered}
$$

and

$$
\operatorname{sur}_{\mathcal{F}}(W) \leqslant 3+(t-2)+(t-4)=2 t-3 .
$$

As in the proof of Claim 4, with $y_{2}$ playing the role of $v_{i}$, for $j \in\{1,2\}$ there exists a set $Y_{j}$ such that $\operatorname{sur}_{\mathcal{F}}\left(Y_{j}\right) \leqslant 2,\left\{u, b_{j}\right\} \subset Y_{j}$ and $y_{2} \notin Y_{j}$. Since $Y_{1} \cap Y_{2} \neq \emptyset$,

$$
\operatorname{sur}_{\mathcal{F}}\left(Y_{1} \cup Y_{2}\right) \leqslant \operatorname{sur}_{\mathcal{F}}\left(Y_{1}\right)+\operatorname{sur}_{\mathcal{F}}\left(Y_{2}\right)-\operatorname{sur}_{\mathcal{F}}\left(Y_{1} \cap Y_{2}\right) \leqslant 2+2-1=3 .
$$

If $w \in Y_{1} \cup Y_{2}$ then $X:=Y_{1} \cup Y_{2}$ will satisfy (4.13). So we may suppose $w \notin Y_{1} \cap Y_{2}$ : in particular, $b_{1} \neq w \neq b_{2}$. 
Let $j \in\{1,2\}$. Let $B_{j}^{\prime \prime}:=B_{j}-y_{2}+w$ and let $\mathcal{H}_{j}^{\prime \prime}$ be the hypergraph with $V\left(\mathcal{H}_{j}^{\prime \prime}\right)=V$ and $\mathcal{E}\left(\mathcal{H}_{j}^{\prime \prime}\right)=\mathcal{E}-B_{j}+B_{j}^{\prime \prime}$. Then $\operatorname{Skel}\left(\mathcal{H}_{j}^{\prime \prime}\right)$ and $\operatorname{Skel}(\mathcal{H})$ have the same number of edges, and the sum of squares of degrees of vertices is at least as large in $\mathcal{H}_{j}^{\prime \prime}$ as in $\mathcal{H}$ (with equality if $w$ is weak), but the weak vertices in $A$ have three different essential neighbours outside $A$ in $\mathcal{H}_{j}^{\prime \prime}$ (namely, $u, w$ and $b_{3-j}$ ), which is impossible in a minimal counterexample to Theorem 10 by Lemma 15 . Thus the Theorem holds for $\mathcal{H}_{j}^{\prime \prime}$. Suppose that $\mathcal{H}_{j}^{\prime \prime}$ has a panchromatic $t$-colouring $\phi$. If $\phi\left(y_{2}\right) \neq \phi\left(b_{j}\right)$, then $\phi$ gives a $t$-colouring of $\operatorname{Skel}(\mathcal{H})$. If $\phi\left(y_{2}\right)=\phi\left(b_{j}\right)$ then we obtain a $t$-colouring of $F$ violating Claim 1 by recolouring $y_{2}$ differently from $b_{1}$ and $b_{2}$; if $\phi\left(y_{2}\right)$ was equal to $\phi(u)$ then we must also recolour $y_{1}$ with $\phi(u)$, which is possible since $\phi(u)=\phi\left(y_{2}\right)=\phi\left(b_{j}\right) \neq \phi(w)$. Thus $\mathcal{H}_{j}^{\prime \prime}$ has no panchromatic $t$-colouring and so is not well spread; that is, there exists $R_{j}^{\prime \prime} \subseteq V\left(\mathcal{H}_{j}^{\prime \prime}\right)$ with nonpositive surplus. Since $\mathcal{H}$ is well spread, $B_{j}^{\prime \prime} \subseteq R_{j}^{\prime \prime}$. Let $R_{j}:=R_{j}^{\prime \prime} \backslash\left(B_{j}^{\prime \prime} \backslash\left\{w, b_{j}\right\}\right)$. Then, as in the proof of Claim $4, \operatorname{sur}_{\mathcal{F}}\left(R_{j}\right) \leqslant 2$ and $\left\{w, b_{j}\right\} \subset R_{j}$.

If $R_{1} \cap Y_{2} \neq \emptyset$, then $\operatorname{sur}_{\mathcal{F}}\left(R_{1} \cap Y_{2}\right)=\operatorname{sur}_{\mathcal{H}}\left(R_{1} \cap Y_{2}\right) \geqslant 1\left(\right.$ since $\left.y_{2} \notin R_{1} \cap Y_{2}\right)$ and so

$$
\operatorname{sur}_{\mathcal{F}}\left(R_{1} \cup Y_{2}\right) \leqslant \operatorname{sur}_{\mathcal{F}}\left(R_{1}\right)+\operatorname{sur}_{\mathcal{F}}\left(Y_{2}\right)-\operatorname{sur}_{\mathcal{F}}\left(R_{1} \cap Y_{2}\right) \leqslant 2+2-1=3 .
$$

Since $\left\{u, w, b_{1}, b_{2}\right\} \subset R_{1} \cup Y_{2}$, we can take $X:=R_{1} \cup Y_{2}$ in (4.13). The result holds similarly if $R_{2} \cap Y_{1} \neq \emptyset$. We may therefore suppose that $R_{1} \cap Y_{2}=$ $R_{2} \cap Y_{1}=\emptyset$. Let $T:=R_{2} \cup Y_{1} \cup Y_{2}$. Then $R_{1} \cap T$ is the disjoint union of nonempty sets $R_{1} \cap Y_{1}$ (containing $b_{1}$ ) and $R_{1} \cap R_{2}$ (containing $w$ ), so that

$\operatorname{sur}_{\mathcal{F}}\left(R_{1} \cap T\right)=\operatorname{sur}_{\mathcal{F}}\left(R_{1} \cap Y_{1}\right)+\operatorname{sur}_{\mathcal{F}}\left(R_{1} \cap R_{2}\right)+(t-2) \geqslant 1+1+t-2=t$.

By (4.14) and since $b_{2} \in R_{2} \cap Y_{2}$,

$\operatorname{sur}_{\mathcal{F}}(T) \leqslant \operatorname{sur}_{\mathcal{F}}\left(R_{2}\right)+\operatorname{sur}_{\mathcal{F}}\left(Y_{1} \cup Y_{2}\right)-\operatorname{sur}_{\mathcal{F}}\left(R_{2} \cap\left(Y_{1} \cup Y_{2}\right)\right) \leqslant 2+3-1=4$, and

$$
\operatorname{sur}_{\mathcal{F}}\left(R_{1} \cup T\right) \leqslant \operatorname{sur}_{\mathcal{F}}\left(R_{1}\right)+\operatorname{sur}_{\mathcal{F}}(T)-\operatorname{sur}_{\mathcal{F}}\left(R_{1} \cap T\right) \leqslant 2+4-t \leqslant 3 .
$$

Since $\left\{u, w, b_{1}, b_{2}\right\} \subset R_{1} \cup T$, we can take $X:=R_{1} \cup T$ in (4.13). This completes the proof of Claim 7 and of Lemma 16.

We will now show that

$$
t|V| \leqslant\left(t^{2}-2 t+2\right)|\mathcal{E}|,
$$

which by (4.1) will imply that $\operatorname{sur}_{\mathcal{H}}(V)<0$, and this contradiction will complete the proof of Theorem 10. 
Give each vertex of $\mathcal{H}$ a "charge" equal to $\left(t^{2}-2 t+2\right)$ times its degree. Then the total charge assigned to all vertices is $t\left(t^{2}-2 t+2\right)|\mathcal{E}|$. We shall now redistribute the charge, without creating or destroying any, and we shall show that after the redistribution every vertex has charge at least $t^{2}$ and every edge has nonnegative charge; this will prove (4.15).

The rules for redistribution are as follows.

Rule 1. Each essential vertex sends $t-1$ to each adjacent 1 -vertex.

Rule 2. Each vertex that is incident with two or more full edges sends $\frac{1}{2} t^{2}-2 t+2$ to each such edge.

Rule 3. Each vertex $v$ that is incident with exactly one full edge and $k$ hollow edges sends to the full edge $\frac{1}{2} t^{2}-2 t+2$ if $v$ is special and $k t-2 t+2$ otherwise. (Note that this is at least $\frac{1}{2} t^{2}-2 t+2$ if $k \geqslant \frac{1}{2} t$.)

Rule 4 . For odd $t$, each special edge $A$ receives $\frac{1}{2} t(t-2)$ from the $A$-special vertex $u(A)$.

Rule 5. Each full edge $A$ gives $t-2$ to every weak vertex in $A$ and (for odd $t)$ gives $t$ to every very special vertex in $A$.

We now check that, after this redistribution of charge, every vertex has charge at least $t^{2}$ and every edge has nonnegative charge.

1. Inessential vertices. Each 1 -vertex $v$ starts with $t^{2}-2 t+2$ and gains $2(t-1)$ by Rule 1 from the two essential vertices in the hollow edge containing $v$. It is not affected by Rules $2-5$, and so it ends up with charge $t^{2}$.

2. Weak vertices. Each weak vertex gives $(t-2)(t-1)$ by Rule 1 and gains $t-2$ by Rule 5 , thereby ending up with $2\left(t^{2}-2 t+2\right)-(t-2)^{2}=t^{2}$.

3. Vertices incident with at least two full edges. Let $v$ be a vertex incident with $r \geqslant 2$ full edges and $k \geqslant 0$ hollow edges. Then $v$ gives $k(t-2)(t-1)$ by Rule $1, r\left(\frac{1}{2} t^{2}-2 t+2\right)$ by Rule 2 and at most $\lfloor k /(t-2)\rfloor \frac{1}{2} t(t-2) \leqslant \frac{1}{2} k t$ by Rule 4. Thus $v$ is left with at least

$$
\begin{gathered}
(r+k)\left(t^{2}-2 t+2\right)-k(t-2)(t-1)-r\left(\frac{1}{2} t^{2}-2 t+2\right)-\frac{1}{2} k t \\
=\frac{1}{2}\left(r t^{2}+k t\right) \geqslant t^{2} .
\end{gathered}
$$

4. Vertices incident with exactly one full edge. We have dealt with weak vertices already, so let $v$ be a vertex incident with one full edge and $k \geqslant 2$ hollow edges. There are three cases.

Case 1. $v$ is not special. Then $v$ gives $k(t-2)(t-1)$ by Rule 1 and $k t-2 t+2$ by Rule 3 and is left with

$$
(1+k)\left(t^{2}-2 t+2\right)-k(t-2)(t-1)-(k t-2 t+2)=t^{2} .
$$


Case 2. $k \leqslant t-2$ and $v$ is special. Then, by the definition of a special vertex, $k=t-2$ and $v$ is very special. Hence $v$ gives $(t-2)^{2}(t-1)$ by Rule $1, \frac{1}{2} t^{2}-2 t+2$ by Rule 3 and $\frac{1}{2} t(t-2)$ by Rule 4 , and receives $t$ by Rule 5 , ending up with

$$
(t-1)\left(t^{2}-2 t+2\right)-(t-2)^{2}(t-1)-\left(\frac{1}{2} t^{2}-2 t+2\right)-\frac{1}{2} t(t-2)+t=t^{2} .
$$

Case 3. $k \geqslant t-1$ and $v$ is special. Then $v$ gives $k(t-2)(t-1)$ by Rule 1 , $\frac{1}{2} t^{2}-2 t+2$ by Rule 3 and at most $\lfloor k /(t-2)\rfloor \frac{1}{2} t(t-2) \leqslant \frac{1}{2} k t$ by Rule 4 . If $k \geqslant t$ then $v$ is left with at least $t^{2}$ by (4.16) with $r=1$. If $k=t-1$ then $v$ ends up with at least

$$
t\left(t^{2}-2 t+2\right)-(t-1)^{2}(t-2)-\left(\frac{1}{2} t^{2}-2 t+2\right)-\frac{1}{2} t(t-2)=t^{2} .
$$

5. Vertices not incident with full edges. We have dealt with inessential vertices already, so let $v$ be an essential vertex incident with no full edges and $k$ hollow edges. By Lemma 13, $k \geqslant t$. There are three cases.

Case 1. $v$ is not special. Then $v$ gives only $k(t-2)(t-1)$ by Rule 1 and is left with

$$
k\left(t^{2}-2 t+2\right)-k(t-2)(t-1)=k t \geqslant t^{2} .
$$

Case 2. $k \geqslant 2 t-2$ and $v$ is special. Then $v$ gives $k(t-2)(t-1)$ by Rule 1 and at most $\lfloor k /(t-2)\rfloor \frac{1}{2} t(t-2) \leqslant \frac{1}{2} k t$ by Rule 4 . If $k \geqslant 2 t$ then $v$ is left with at least

$$
k\left(t^{2}-2 t+2\right)-k(t-2)(t-1)-\frac{1}{2} k t=\frac{1}{2} k t \geqslant t^{2} .
$$

If $k=2 t-1$ or $2 t-2$ then $v$ is left with at least

$$
k\left(t^{2}-2 t+2\right)-k(t-2)(t-1)-t(t-2)=t(k-t+2) \geqslant t^{2} .
$$

Case 3. $k \leqslant 2 t-3$ and $v$ is special. Since $v$ is special, $t$ is odd and so $t \geqslant 7$. (In fact, it is enough here that $t>3$.) By (s3) in the definition of a special vertex, $k=2 t-3$. If $v$ is special for only one edge $A$ then $v$ is left with at least

$$
k\left(t^{2}-2 t+2\right)-k(t-2)(t-1)-\frac{1}{2} t(t-2)=\frac{3}{2} t^{2}-2 t>t^{2} .
$$

So suppose that there exist distinct edges $A$ and $B$ that are $\left(v, w_{1}, z_{1}\right)$-special and $\left(v, w_{2}, z_{2}\right)$-special respectively. Let $\mathcal{H}^{\prime}$ be the hypergraph obtained from $\mathcal{H}$ by deleting $v$ and all inessential vertices adjacent to $v$. By the minimality of $\mathcal{H}$, there is a panchromatic $t$-colouring of $\mathcal{H}^{\prime}$. Give $v$ a colour different from the colours of $w_{1}, w_{2}$ and $w_{3}$, where $w_{3}$ is the essential vertex adjacent to $v$ and not in $A \cup B$. Now the colouring can easily be extended to the rest of $\mathcal{H}$, a contradiction. 
6. Special edges. Let $A$ be $(u, w, z)$-special. By Lemma $16, z$ is $(t-1)$-full (and hence $\left(\frac{1}{2} t\right)$-full), and so $A$ receives at least $\frac{1}{2} t^{2}-2 t+2$ from $z$ by Rule 2 or Rule 3 and $\frac{1}{2} t(t-2)$ from $u$ by Rule 4 . It also gives $(t-1)(t-2)$ to its $t-1$ weak vertices by Rule 5 , and so ends up with at least 0 . (Observe that $z$ is not very special, since a very special vertex is not $(t-1)$-full; so $A$ gives nothing to $z$ by Rule 5 .)

7. Nonspecial full edges Let $A$ be a full edge containing exactly $m$ weak vertices and $k$ very special vertices, where $m+k \leqslant t$. The net effect of Rules 3 and 5 is that every very special vertex gives $A$ the amount

$$
\left(\frac{1}{2} t^{2}-2 t+2\right)-t=\frac{1}{2}\left(t^{2}-6 t+4\right) \geqslant 5 \frac{1}{2},
$$

since if there exist special vertices then $t$ is odd and so $t \geqslant 7$. A vertex that is neither weak nor very special gives $A$ either $\frac{1}{2} t^{2}-2 t+2 \geqslant 2$ or $k t-2 t+2 \geqslant 2$ by Rule 3 , since in this case $t \geqslant 4$. Thus every nonweak vertex gives $A$ at least 2 . By Rule 5 , the weak vertices collectively take from $A$ exactly $m(t-2)$. So if $m \leqslant 2$ then $A$ ends up with nonnegative charge. Since $A$ is not special, $m \leqslant t-2$. So we may suppose that $3 \leqslant m \leqslant t-2$. There are three cases.

Case 1. There are two vertices in $A$ that are $\left(\frac{1}{2} t\right)$-full and not very special. Together they give $A$ at least $t^{2}-4 t+4 \geqslant m(t-2)$ by Rule 2 or Rule 3 , which is enough.

Case 2. At most one vertex in $A$ that is not very special is $\left(\frac{1}{2} t\right)$-full, and alternative (i) in Lemma 16 holds. Then $m<\frac{1}{2} t$ and the two $m$-full vertices from (i) give $A$ at least $2(m t-2 t+2)$. Since every other nonweak vertex gives $A$ at least $2, A$ ends up with at least

$$
2(m t-2 t+2)+2(t-m-2)-m(t-2)=t(m-2)>0 .
$$

Case 3. At most one vertex in $A$ that is not very special is $\left(\frac{1}{2} t\right)$-full, and alternative (ii) in Lemma 16 holds. Then for some $j(3 \leqslant j \leqslant t-m)$ there exist $j$ vertices in $A$ that are not very special and are $(t-j+1)$-full. It follows that $t-j+1<\frac{1}{2} t$, and these vertices give $A$ at least $j((t-j+1) t-2 t+2)$. Since $m \leqslant t-j, A$ ends up with at least

$$
\begin{aligned}
j((t-j+1) t-2 t+2)-m(t-2) & \geqslant j((t-j) t-(t-2))-(t-j)(t-2) \\
& =(j(t-j)-(t-2)) t \\
& =((j-1)(t-1-j)+1) t>t>0 .
\end{aligned}
$$

8. Hollow edges Hollow edges never have any charge, and so we do not need to consider them.

The proof of Theorem 10 is finally complete. 


\section{References}

[1] M. I. Burstein: Critical hypergraphs with minimal number of edges, Bull. Acad. Sci. Georgian SSR, 83 (1976), 285-288 [in Russian].

[2] P. Duchet: Hypergraphs, in: Handbook of Combinatorics (R. L. Graham, M. Grötschel and L. Lovász eds.), Elsevier, Amsterdam, 1995, 381-432.

[3] T. Gallai: Kritische Graphen I, Publ. Math. Inst. Hungar. Acad. Sci., 8 (1963), $165-192$.

[4] P. Hall: On representatives of subsets, J. London Math. Soc., 10 (1935), 26-30.

[5] M. Krivelevich: On the minimal number of edges in color-critical graphs, Combinatorica, 17 (1997), 401-426.

[6] L. Lovász: A generalization of König's theorem, Acta Math. Acad. Sci. Hungar., 21 (1970), 443-446.

[7] P. D. Seymour: On the two-colouring of hypergraphs, Quart. J. Math., 25 (1974), 303-312.

[8] D. R. Woodall: Property B and the four-colour problem, in: Combinatorics (Institute of Mathematics and its Applications, Southend-on-sea, England, 1972), 322-340.

Alexandr V. Kostochka

Institute of Mathematics, Novosibirsk, 630090, Russia and

Department of Mathematics

University of Illinois at Urbana-Champaign

Urbana, IL 61801, USA

kostochk@math.uiuc.edu

\section{Douglas R. Woodall}

School of Mathematical Sciences, University of Nottingham, Nottingham, NG7 2RD, England douglas. woodall@nottingham.ac.uk 